\title{
Evaluation of Solid-Supported Room-Temperature Ionic Liquids Containing Crown Ethers as Media for Metal Ion Separation and Preconcentration
}

\author{
Cory A. Hawkins ${ }^{\mathrm{a}, \mathrm{b}}$, M. A. Momen ${ }^{\mathrm{a}}$, Sarah L. Garvey ${ }^{\mathrm{a}}$, John Kestell ${ }^{\mathrm{a}}$, \\ Michael D. Kaminski ${ }^{\mathrm{b}}$, and Mark L. Dietz ${ }^{\mathrm{a}}{ }^{\text {, }}$ * \\ ${ }^{a}$ Department of Chemistry and Biochemistry, University of Wisconsin-Milwaukee, \\ 3210 N. Cramer Street, Milwaukee, WI 53211, United States \\ ${ }^{\mathrm{b}}$ Chemical Sciences and Engineering Division, Argonne National Laboratory, \\ 9700 S. Cass Avenue, Argonne, IL 60439, United States
}

\begin{abstract}
Extraction chromatographic (EXC) resins incorporating an appropriate crown ether in an oxygenated organic solvent such as 1-octanol are well established as sorbents for the analyticalscale separation and preconcentration of radiostrontium from a variety of sample types. Recent solvent extraction studies employing crown ethers in various 1-alkyl-3-methylimidazolium-based $\left(\mathrm{C}_{\mathrm{n}} \mathrm{C}_{1} \mathrm{im}^{+}\right)$room-temperature ionic liquids (RTILs) indicate that under certain conditions, distribution ratios $\left(\mathrm{D}_{\mathrm{Sr}}\right)$ for strontium far in excess of those observed with conventional organic solvents are observed. To determine if this increase in liquid-liquid extraction efficiency will lead to improved strontium sorbents, several EXC resins and sol-gel glasses incorporating ditert-butylcyclohexano-18-crown-6 (DtBuCH18C6) in either 1-decyl-3-methylimidazolium bis[(trifluoromethyl)sulfonyl]imide $\left(\mathrm{C}_{10} \mathrm{C}_{1} \mathrm{imTf}_{2} \mathrm{~N}\right)$ or the related hydroxyalkyl-functionalized IL 1-(12-hydroxydodecyl)-3-butylimidazolium $\quad$ bis[(trifluoromethyl)sulfonyl]imide $\quad\left(\mathrm{C}_{12} \mathrm{OHC}_{4} \mathrm{im}\right.$ $\mathrm{Tf}_{2} \mathrm{~N}$ ) were prepared and characterized. Unexpectedly the performance of these materials was not uniformly better than that of a conventional EXC resin, an apparent result of the greater viscosity of the ionic liquids and the lower solubility of the crown ethers in ILs versus conventional organic solvents.
\end{abstract}

Keywords: Extraction chromatography, ionic liquids, crown ethers, strontium

* To whom correspondence should be addressed. E-mail address: dietzm@uwm.edu. Tel: 1-414-229-1748; Fax: 1-414-229-5530. 


\section{Introduction}

Extraction chromatography, a type of liquid chromatography in which the stationary phase comprises a metal ion extractant or a solution of the extractant in an appropriate diluent sorbed on an inert (often, polymeric) support, has proven to be of extraordinary utility in the development of methodology for the determination of a variety of radionuclides in environmental (e.g., soil) and biological (e.g., bioassay) samples (1-3). Despite its many advantages, however, extraction chromatography suffers from several significant limitations (24). First, because the extractant is sorbed rather than covalently bonded to the support, the physical stability of extraction chromatographic (EXC) resins can be inadequate for many applications. In addition, the capacity of typical EXC sorbents is rather limited, often to only a few $\mathrm{mg} / \mathrm{mL}$ of bed volume. As a result, inconveniently large columns may be required if extractant saturation (i.e., column overload) is to be avoided. Finally, the retention of metal ions on certain EXC materials is often insufficient, even in the absence of competing ions that might be expected to consume a significant fraction of the sorption sites on the material. For example, although an EXC resin selective for radiostrontium (5-7) has long been commercially available (i.e., Sr resin, EiChroM Technologies), its modest strontium retention makes it poorly suited for large-volume samples and complicates efforts to miniaturize chromatographic separations employing it.

Prior work has shown that metal ion extraction by crown ethers such as that on which the commercial strontium resin is based (di-tert-butylcyclohexano-18-crown-6, hereafter abbreviated as DtBuCH18C6) is strongly influenced by the organic solvent in which the extractant is dissolved $(8,9)$. For example, strontium extraction into a solution of dicyclohexano-18-crown-6 (DCH18C6) and by analogy, its retention on a crown ether-based EXC resin $(5,10)$, has been found to increase as the molecular weight of the solvent is decreased within a given diluent family, the apparent result of the greater solubility of water in lower molecular weight solvents and the accompanying increase in the ease of solvating the co-extracted anion (8). As a compromise between the need to maximize strontium retention and to minimize the loss of the crown ether/diluent mixture comprising the stationary phase to solubilization in the mobile phase during a separation, 1-octanol is employed as the stationary phase solvent in the commercial resin $(5,6)$. Recent work suggests that better results, in particular far stronger retention of strontium, might be achieved by employing ionic liquids (ILs), a novel class of solvents typically 
comprising a bulky, asymmetric organic cation in combination with any of a wide variety of organic or inorganic anions (11) as the basis of the stationary phase. Specifically, various studies have shown that under certain conditions, metal ion extraction efficiencies far exceeding those seen with conventional, molecular solvents can be obtained in liquid-liquid systems employing solutions of an appropriate extractant in any of a variety of ILs (12-14). For example, using solutions of DCH18C6 in N,N'-dialkylimidazolium-based ILs, Dai et al. (12) have demonstrated that strontium distribution ratios $\left(\mathrm{D}_{\mathrm{Sr}}\right.$, defined as the ratio of the total concentration of strontium in the organic phase to that in the aqueous phase at equilibrium, $\left.[\mathrm{Sr}]_{\mathrm{org}, \mathrm{eq}} /[\mathrm{Sr}]_{\mathrm{aq}, \mathrm{eq}}\right) 10^{4}$ times larger than those observed in 1-octanol can be obtained. This suggests that an EXC resin employing an appropriate IL as a component of the stationary phase may provide strontium ion retention significantly greater than that obtained with conventional EXC resins.

In the past decade, a number of metal ion sorbents have been described which comprise an ionic liquid dispersed in/on a solid support (15-25). Much less common are those in which an ionic liquid is employed as the diluent for a supported metal ion extractant (26-30). In this report, we describe the preparation and preliminary characterization of several strontium sorbents incorporating a mixture of a crown ether and various $N, N$ '-dialkylimidazolium-based ILs, supported in either a porous polymer matrix, as is often done in extraction chromatography, or a sol-gel glass. Unexpectedly the results obtained indicate that the performance, in particular the strontium retention, of these new materials is not necessarily superior to that of the conventional commercial EXC resin.

\section{Experimental}

\section{Materials and Methods}

Reagents. Dicyclohexano-18-crown-6 (DCH18C6) was obtained as a mixture of the cissyn-cis (A) and cis-anti-cis (B) isomers from Parish Chemical Company (Orem, UT). The 4,4'(5')-di-(tert-butylcyclohexano)-18-crown-6 (DtBuCH18C6) was obtained from EichroM Technologies, Inc. (Darian, IL) and used without further purification. Amberchrom ${ }^{\mathrm{TM}}$ CG-71m was purchased from Rohm and Haas (Philadelphia, PA) and pretreated as previously described (31). The tetramethyl orthosilicate (TMOS) and 1-octanol were obtained from Alfa Aesar (Heysham, UK), while the formic acid (98\%) was obtained from Sigma-Aldrich (St. Louis, MO). All were used as received. Optima ${ }^{\mathrm{TM}}$ grade nitric acid and HPLC grade methanol were obtained 
from Fisher Scientific Company (Waltham, MA). The Sr-85 and Na-22 radiotracers were purchased from Perkin Elmer Corporation (Shelton, CT) as the respective chlorides and converted to the nitrate form by repeated evaporation to dryness in the presence of nitric acid. All water was obtained from a Milli-Q2 system and had a specific resistance of at least $18 \mathrm{M} \Omega$ $\mathrm{cm}$. The ionic liquids employed in this work, 1-decyl-3-methylimidazolium bis[(trifluoromethyl)sulfonyl]imide $\quad\left(\mathrm{C}_{10} \mathrm{C}_{1} \mathrm{imTf}_{2} \mathrm{~N}\right) \quad$ and 1-(12-hydroxydodecyl)-3butylimidazolium bis[(trifluoromethyl)sulfonyl]imide $\left(\mathrm{C}_{12} \mathrm{OHC}_{4} \mathrm{imTf}_{2} \mathrm{~N}\right)$, were prepared by reaction of the corresponding bromides with $\operatorname{LiTf}_{2} \mathrm{~N}$ and purified using methods described previously $(11,32,33)$. Sample purity was verified by ${ }^{1} \mathrm{H}-$ and ${ }^{13} \mathrm{C}-\mathrm{NMR}$ (DMSO), as detailed in previous reports $(11,32,33)$. The NMR spectra were acquired on a Bruker DPX300 NMR spectrometer operating at $300.13 \mathrm{MHz}$ for proton and $75.47 \mathrm{MHz}$ for carbon-13, and equipped with a z-gradient broadband (BBO) probe. Spectra were obtained using solutions in dimethylsulfoxide- $\mathrm{d}_{6}$ (Aldrich, 99.96 atom\% D), and all chemical shifts were reported relative to tetramethylsilane.

Procedures. Liquid-liquid extraction studies. The distribution of the radiotracer between the aqueous and organic phases of interest was determined by equilibrating equal volumes of a tracer-spiked nitric acid solution and either 1-octanol or an ionic liquid. Prior to the distribution measurement, the organic phase was pre-equilibrated via two contacts with twice its volume of an appropriate acid solution. From the measured activity of the aqueous and organic phases after equilibration, determined via gamma spectroscopy according to standard procedures on a PerkinElmer 2480 Automatic Gamma Counter, the distribution ratio of strontium (or sodium) was calculated from the equation:

$$
\mathrm{D}_{\mathrm{M}}=[\mathrm{M}]_{\mathrm{org}, \mathrm{eq}} /[\mathrm{M}]_{\mathrm{aq}, \mathrm{eq}}
$$

Polymer-supported IL-crown ether mixtures. The EXC resins comprising polymersupported IL-crown ether mixtures were prepared in a manner analogous to that described previously for mixtures of crown ethers and conventional organic solvents $(5,6)$.

Sol-gel-encapsulated IL-crown ether mixtures. To synthesize the silica-based EXC materials, an acid-catalyzed sol-gel procedure was employed (34). In a typical preparation, DtBuCH18C6 (50 mg) was dispersed into a mixture of TMOS (1 mL) and formic acid (2 mL). This route yielded a "solvent-less" (i.e., no diluent added) sorbent. To determine the impact of the presence of a solvent, the same route for preparation was followed, but neat DtBuCH18C6 
was replaced with a $1 \mathrm{M}$ solution of the crown ether in either the IL or 1-octanol. For all preparations, the quantity of DtBuCH18C6 used was maintained at $50 \mathrm{mg}$. After a week of standing, during which time gelation and the volatilization of the $\mathrm{CH}_{3} \mathrm{OH}$ and $\mathrm{HCOOCH}_{3}$ hydrolysis products occurred, a monolithic glass composite consisting of DtBuCH18C6 or its solution in the IL or 1-octanol entrapped in the silica network (35) was obtained. The composite glass material was crushed and sieved to collect material of the desired size range $(\sim 50-100 \mu \mathrm{m}$ diameter) for subsequent metal ion uptake experiments. Following sieving, the ground material was examined by scanning electron microscopy using a Hitachi Model S-4800 field emission SEM.

Sorbent characterization. Equilibrium metal ion uptake. Solid-liquid (weight) distribution ratios $\left(D_{\mathrm{w}}\right)$ for strontium and sodium were determined radiometrically using ${ }^{85} \mathrm{Sr}$ and ${ }^{22} \mathrm{Na}$ radiotracers. Specifically, the sorption of the tracer from nitric acid solutions by the resins was measured by contacting a known volume (typically $1.0 \mathrm{~mL}$ ) of tracer-spiked acid solution of appropriate concentration with a known mass of resin. The ratio of the aqueous phase volume $(\mathrm{mL})$ to the weight $(\mathrm{g})$ of the chromatographic material ranged from 40-50. (This ratio is determined primarily by the need to produce an easily measured decrease in the aqueous activity by contact with the resin.) Contact times of two and four hours (with occasional swirling) were employed for the polymeric and silica-based EXC materials, respectively. After equilibration, an aliquot of the aqueous phase was withdrawn from each culture tube and filtered through a 0.22 $\mu \mathrm{m}$ poly(vinylidene fluoride) (PVDF) filter to ensure that no dispersed resin was present. On the basis of the initial and final activity of a measured aliquot of this aqueous phase, the weight distribution ratio of strontium or sodium was calculated from the following equation:

$$
D_{w}=\left[\left(A_{0}-A_{f}\right) / A_{f}\right](V / w)
$$

where $A_{0}$ and $A_{f}$ represent the aqueous phase activity (cpm) before and after equilibration respectively, $w$ is the mass of the resin taken $(\mathrm{g})$, and $\mathrm{V}$ is the volume of the aqueous phase $(\mathrm{mL})$.

Sorbent characterization. Metal ion uptake kinetics. Into a series of screw-cap test tubes, each containing the same amount $(20 \pm 2 \mathrm{mg})$ of EXC resin, was introduced a known volume (typically $1 \mathrm{~mL}$ ) of an appropriate nitric acid solution containing a $\mathrm{Sr}-85$ radiotracer. At various time intervals following the introduction of the tracer solution, during which the samples were periodically mixed to ensure equilibration, the aqueous phase was withdrawn from one of the test tubes and filtered through a $0.22 \mu \mathrm{m}$ PVDF filter. From the initial and final activity of the 
aqueous phases, $D_{w}$ values were determined as described above and a plot of the time dependence of $\mathrm{D}_{\mathrm{w}}$ was prepared.

\section{Results and Discussion}

Liquid-liquid extraction studies. In an earlier report, Horwitz and Dietz (36) examined the relationship between the efficiency with which a given metal ion is extracted in a liquidliquid (1-1) system, as reflected in the metal ion distribution ratio $\left(\mathrm{D}_{\mathrm{M}}\right)$ and its retention on an extraction chromatographic sorbent based on this 1-1 system, as reflected in the weight distribution ratio for the ion. For lanthanide ( $\mathrm{Ln}$ ) extraction by several organophosphorus acids, it was found that despite some differences between the measured values of the capacity factor, $\mathrm{k}$, for the Ln ions and those expected on the basis of $\mathrm{D}_{\mathrm{M}}$ measurements (given by $\mathrm{D}_{\mathrm{M}} \cdot \mathrm{V}_{\mathrm{s}} / \mathrm{V}_{\mathrm{m}}$, where $V_{s}$ and $V_{m}$ are the stationary and mobile phase volumes, respectively), the overall trend of decreasing metal ion affinity for the organic phase with rising acidity was generally the same in both the 1-1 and EXC systems. On the basis of this and similar observations by a number of other investigators on a variety of extraction systems (37-44), it is now well established that the extraction behavior of a metal ion is often a useful predictor of its retention behavior on the corresponding EXC resin. For this reason, our efforts to develop an ionic liquid-based EXC material for strontium began with a consideration of the extraction behavior of strontium into a series of ILs incorporating DCH18C6 or its di-tert-butyl- analog, compounds known to exhibit substantial affinity for $\mathrm{Sr}^{2+}$ in conventional solvent systems (45-47).

Figure 1 shows the nitric acid dependency of the extraction of strontium ion by DCH18C6 into two ionic liquids, one alkyl-substituted $\left(\mathrm{C}_{10} \mathrm{C}_{1} \operatorname{imTf}_{2} \mathrm{~N}\right)$ and the other hydroxylfunctionalized $\left(\mathrm{C}_{12} \mathrm{OHC}_{4} \mathrm{imTf}_{2} \mathrm{~N}\right)$. Also shown for purposes of comparison are the results obtained under the same conditions using a conventional molecular diluent, 1-octanol, as the organic solvent. In addition, the corresponding results for $\mathrm{Na}^{+}$extraction into both 1-octanol and $\mathrm{C}_{10} \mathrm{C}_{1} \mathrm{imTf}_{2} \mathrm{~N}$ are provided. As can be seen, extraction into the molecular diluent follows the expected trend (48) of generally increasing extraction efficiency $\left(\mathrm{D}_{\mathrm{M}}\right)$ with rising aqueous nitrate concentration. Note that at sufficiently high acidities, competition for the extractant between the acid present and the metal ion (49) eventually leads to a downturn in the acid dependency for sodium ion. The net result is an increase in the strontium/sodium separation factor, $\alpha_{\mathrm{Sr} / \mathrm{Na}}$, with acidity. For the ionic liquids also, strontium extraction generally rises with increasing aqueous 
acidity, indicating as noted in prior studies (14) that ion-pair extraction/extraction of a neutral strontium-crown ether-nitrato complex is the predominant mode of extraction under the experimental conditions. In contrast, the extraction of sodium ion into $\mathrm{C}_{10} \mathrm{C}_{1} \mathrm{imTf}_{2} \mathrm{~N}$ declines over the entire range of acidities examined, behavior consistent with the predominance of ionexchange in the overall extraction process $(14,50)$. In this extraction system too then, $\alpha_{\mathrm{Sr} / \mathrm{Na}}$ varies with acidity, in this case peaking at $c a .3 \mathrm{M} \mathrm{HNO}_{3}$, the acidity most commonly employed for sample loading in procedures incorporating commercial resins for strontium sorption, at a value $c a$. 64, approximately three times that obtained in 1-octanol (51). These results, together with the higher values of $\mathrm{D}_{\mathrm{Sr}}$ observed in the 1-3 $\mathrm{M} \mathrm{HNO}_{3}$ range, suggest that it should be possible to significantly improve the performance of established commercial strontium sorbents simply by replacing the diluent employed (1-octanol) with an appropriate ionic liquid.

Because the not insignificant water solubility of DCH18C6 (52) would be expected to make an EXC material prepared from it unstable, its more hydrophobic di-tert-butyl-substituted analog, DtBuCH18C6, was next evaluated. As has been noted previously (53), this compound can exist in numerous isomeric forms. To facilitate the interpretation of the extraction data, we have employed a single form, designated as the 4z,5'z-cis-syn-cis isomer (Figure 2), believed to be one of the major constituents of commercial preparations of this crown ether (54). Figure 3 depicts the nitric acid dependency of $\mathrm{D}_{\mathrm{Sr}}$ for this compound, again in 1-octanol, $\mathrm{C}_{10} \mathrm{C}_{1} \mathrm{imTf}_{2} \mathrm{~N}$, and $\mathrm{C}_{12} \mathrm{OHC}_{4} \mathrm{imTf}_{2} \mathrm{~N}$, along with analogous results for sodium ion in 1-octanol and $\mathrm{C}_{10} \mathrm{C}_{1} \mathrm{imTf}_{2} \mathrm{~N}$. In the conventional molecular diluent (i.e., 1-octanol), the dependency for both $\mathrm{Sr}$ and $\mathrm{Na}$ extraction generally exhibits the increase with rising acidity expected for neutral complex extraction, and the $\mathrm{Sr} / \mathrm{Na}$ separation factor $\left(\alpha_{\mathrm{Sr} / \mathrm{Na}}\right)$ approaches 100 at the highest acidities $(c a .6$ $\mathrm{M})$. For the ionic liquids, $\mathrm{D}_{\mathrm{Sr}}$ again generally increases with aqueous acidity, although some flattening or downturn in the dependency is observed above ca. $2 \mathrm{M} \mathrm{HNO}_{3}$. At acidities greater than $c a$. $0.25 \mathrm{M}$, the value of $\mathrm{D}_{\mathrm{Sr}}$ is typically a factor of 5-10 greater than that obtained with DCH18C6, as would be expected from the greater hydrophobicity of the di-t-butyl-substituted compound. Equally important is that strontium-sodium separation factors of 50-100 are observed in $\mathrm{C}_{10} \mathrm{C}_{1} \mathrm{imTf}_{2} \mathrm{~N}$ for 1-6 $\mathrm{M} \mathrm{HNO}_{3}$. Overall then, the use of the more hydrophobic crown ether yields strontium extraction efficiencies significantly higher than those seen with DCH18C6, while preserving its excellent strontium extraction selectivity. In addition, when combined with an IL, DtBuCH18C6 provides $\mathrm{D}_{\mathrm{Sr}}$ values much greater than those seen with 1- 
octanol. Taken together, these results further reinforce the notion that a substantial improvement in the performance of an EXC material for strontium might be effected by use of an ionic liquid diluent.

Polymer-supported crown ether-ionic liquid mixtures. Metal ion uptake studies. To investigate this possibility, extraction chromatographic resins incorporating $40 \%(\mathrm{w} / \mathrm{w})$ of a 1.0 $\mathrm{M}$ solution of DtBuCH18C6 in 1-octanol, $\mathrm{C}_{10} \mathrm{C}_{1} \mathrm{imTf}_{2} \mathrm{~N}$ or $\mathrm{C}_{12} \mathrm{OHC}_{4} \mathrm{imTf}_{2} \mathrm{~N}$ were prepared and evaluated for strontium uptake. Figure 4 shows the nitric acid dependence of strontium uptake (as reflected in the weight distribution ratio, $\mathrm{D}_{\mathrm{w}}$ ) by the three resins. It is immediately apparent that contrary to expectations, the IL-based resins actually provide poorer retention of $\mathrm{Sr}^{2+}$ than does the conventional (i.e., commercial) octanol-based material in the acidity range of interest (ca. 1-6 M). In addition, and again contrary to results observed in the 1-1 extraction studies, there is little difference between strontium retention by materials based on the dialkyl- and hydroxyalkyl-ILs. Thus in contrast to the conventional solvent system (i.e., 1-octanol), for which it has been shown that solvent extraction and extraction chromatographic behavior are reasonably well correlated $(5,6,48), 1-1$ extraction data for the ionic liquids considered here are seemingly of little value in predicting the behavior of the EXC resins prepared from them.

Effects of stationary phase viscosity. In principle, if the partitioning mechanism for a metal ion in a liquid-liquid extraction system and the corresponding extraction chromatographic resin are the same, then the adaptation of the solvent extraction system to a solid support should be straightforward (55). There are, however, several additional issues that must be considered when attempting to understand the relationship between the behavior of the two systems for the ILs, among them the effect of solvent viscosity. According to the Stokes-Einstein Equation, the diffusion coefficient of a solute in a medium is inversely proportional to the viscosity of the medium:

$$
\mathrm{D}=\mathrm{k}_{\mathrm{B}} \mathrm{T} / 6 \pi \eta \mathrm{r}
$$

where $\mathrm{D}$ is the diffusion coefficient of a spherical particle, $\mathrm{k}_{\mathrm{B}}$ is Boltzmann's constant, $\mathrm{T}$ is the absolute temperature, $\eta$ is the viscosity, and $r$ is the radius of the particle (56). As applied to extraction chromatography, this equation indicates that the rate of diffusion of a solute (e.g., strontium ion) into the pores of the EXC resin beads will be reduced as the viscosity of the solution with which the resin has been impregnated increases. In addition, it suggests that the accessibility of extractant molecules within the pores of a support may be limited when the 
viscosity of the stationary phase is high. That is, for a solute to interact with an extractant, a path for solute/extractant transport must be provided. If providing such a path requires that relatively large IL molecules rotate or translate, however, the high viscosity of the medium could render this difficult, thus restricting the availability of the extractant to the solute. For 1-octanol, a viscosity of $7.2 \mathrm{cP}$ has been reported at standard temperature and pressure (57), while for typical ionic liquids, viscosities of 300-1000 cP are observed under the same conditions (58). Clearly then the differences in viscosity of the two solvents are substantial, and thus may at least partially account for the observations.

Extractant concentration effects. The effect of extractant concentration must also be considered. That is, while the solvent extraction experiments described here were carried out using a $0.1 \mathrm{M}$ solution of DtBuCH18C6, the stationary phase of the EXC resin comprises a $1 \mathrm{M}$ solution of the same extractant, a factor of ten more concentrated. Table 1 summarizes the results of 1-1 extraction experiments in which the dependence of the distribution ratio of strontium on the concentration of DtBuCH18C6 in either $\mathrm{C}_{10} \mathrm{C}_{1} \mathrm{imTf}_{2} \mathrm{~N}$ or 1-octanol was determined. As can be seen, in the conventional solvent, an increase in extractant concentration is accompanied by a (roughly) proportional increase in the value of $\mathrm{D}_{\mathrm{Sr}}$. A log-log plot of $\mathrm{D}_{\mathrm{Sr}} v s$. [DtBuCH18C6], in fact, yields a line of near-unit slope (0.78). As also shown in the table, this increase in extractant concentration is obviously accompanied also by a decrease in the mole ratio of solvent to extractant. Even at the highest DtBuCH18C6 concentration, however, a significant excess of the solvent remains. For the ionic liquid, however, neither of these observations applies. That is, as the extractant concentration is increased, $\mathrm{D}_{\mathrm{Sr}}$ initially increases, but then declines. Moreover, in the most concentrated DtBuCH18C6 solutions, the very high molar mass of the ionic liquid means that the solvent-to-extractant mole ratio is quite low, falling to only 1.2 at $1 \mathrm{M}$ extractant. Thus, in these solutions, the system is rapidly approaching one in which the ionic liquid is no longer the majority component. This dearth of solvent apparently has a destabilizing effect, as upon standing in contact with an acidic aqueous phase, the crown ether precipitates from the most concentrated solutions, thus limiting the solubility of DtBuCH18C6 to $c a$. $0.6 \mathrm{M}$. This strongly suggests that precipitation of the extractant in the pores of the support may be at least partly responsible for the unexpectedly poor performance of the IL-based EXC resins. 
It is both interesting and somewhat unexpected to note that precipitation of the extractant from the hydroxyl-functionalized $\mathrm{IL} \mathrm{C}_{12} \mathrm{OHC}_{4} \mathrm{imTf}_{2} \mathrm{~N}$, which by design, bears resemblance to an aliphatic alcohol, occurs to essentially the same extent observed for $\mathrm{C}_{10} \mathrm{C}_{1} \mathrm{imTf}_{2} \mathrm{~N}$ (albeit more slowly) when the solution is allowed to stand in contact with an acidic aqueous phase (e.g., $1 \mathrm{M}$ $\mathrm{HNO}_{3}$ ). That acid contact induces precipitation in this system as well indicates that formation of an insoluble crown ether-hydronium ion adduct is likely responsible. Such precipitation is not without precedent; in fact, the precipitation of a $\mathrm{DtBuCH} 18 \mathrm{C} 6-\mathrm{H}_{3} \mathrm{O}^{+}$adduct from $n$-hexane, induced by perchloric acid contact, has been employed as a means of purifying DtBuCH18C6 (53). Moreover, although seemingly vastly different than hexane, ionic liquids bearing long alkyl chains (as is the case for both ILs considered here) have been found to form nanostructured domains in which the polar head groups and hydrophobic side chains are arranged in such a way as to provide regions differing greatly in polarity (59-61). Here the hydrophobic domains would likely resemble a long-chain alkane, not unlike hexane.

It is also worth noting here that over a short time frame (i.e., minutes to hours), solutions of DtBuCH18C6 in $\mathrm{C}_{10} \mathrm{C}_{1} \mathrm{imTf}_{2} \mathrm{~N}$ more concentrated than its apparent equilibrium solubility can be prepared and studied. As shown in Table 1, for example, strontium distribution ratios can be measured for even a $0.75 \mathrm{M}$ solution of $\mathrm{DtBuCH} 18 \mathrm{C} 6$ with no sign of precipitation over the time span of the measurement. Curiously, however, $\mathrm{D}_{\mathrm{Sr}}$ values at these higher concentrations are lower than those observed at lesser crown ether concentrations. In fact, the extraction of strontium by a $0.75 \mathrm{M}$ solution of the crown ether is essentially identical to that seen at $0.40 \mathrm{M}$ extractant. In an effort to understand the source of this anomaly, the extraction of strontium by DCH18C6, which is not plagued by such solubility limitations, into the same IL was revisited. Figure 5A shows the dependence of $\mathrm{D}_{\mathrm{Sr}}$ and $\mathrm{D}_{\mathrm{Na}}$ on the concentration of DCH18C6 in $\mathrm{C}_{10} \mathrm{C}_{1} \mathrm{imTf}_{2} \mathrm{~N}$ at a fixed aqueous acidity (3.00 $\mathrm{M} \mathrm{HNO}_{3}$ ). For purposes of comparison, the corresponding results for 1-octanol are provided in Figure 5B. As can be seen, in the conventional solvent, $\mathrm{D}_{\mathrm{M}}$ for the monovalent cation (i.e., $\mathrm{Na}^{+}$) increases linearly with rising extractant concentration, an observation consistent with extraction of a neutral sodium nitratocrown ether complex, a process depicted in Equation 1 (with $\mathrm{n}=1$ ):

$$
\mathrm{M}^{\mathrm{n}+}{ }_{\mathrm{aq}}+\mathrm{DCH}_{18 \mathrm{C} 6}{ }_{\text {org }}+\mathrm{n} \mathrm{NO}_{3}^{-}{ }_{\mathrm{aq}} \leftrightarrow \mathrm{M}\left(\mathrm{NO}_{3}\right)_{\mathrm{n}} \cdot \mathrm{DCH} 18 \mathrm{C} 6_{\text {org }}
$$


For the divalent cation (i.e., $\mathrm{Sr}^{2+}$ ), in contrast, significant flattening of the dependency is observed, although $\mathrm{D}_{\mathrm{Sr}}$ values do continue to increase with [DCH18C6], a consequence (as has been described in detail previously (49)) of appreciable aqueous phase complex formation in the strontium-DCH18C6 system.

For the extraction of sodium into the IL, increasing DCH18C6 concentration again yields a proportional increase in $\mathrm{D}_{\mathrm{M}}$. In this instance, however, extraction is believed to proceed predominantly via two types of ion-exchange processes (Figure 6), one in which the sodiumcrown ether complex initially formed is exchanged for the cationic component of the IL, and the other a two-step process involving the initial formation of a 1:1 hydronium ion-DCH18C6 complex and the subsequent exchange of the complexed $\mathrm{H}_{3} \mathrm{O}^{+}$for $\mathrm{Na}^{+}(50)$, as depicted in Equations 2 and 3:

$$
\begin{gathered}
\mathrm{H}_{3} \mathrm{O}^{+}{ }_{\text {aq }}+\mathrm{DCH} 18 \mathrm{C} 6_{\mathrm{IL}} \leftrightarrow \mathrm{DCH} 18 \mathrm{C} 6 \cdot \mathrm{H}_{3} \mathrm{O}^{+}{ }_{\mathrm{IL}} \\
\mathrm{Na}^{+}{ }_{\mathrm{aq}}+\mathrm{DCH} 18 \mathrm{C} 6 \cdot \mathrm{H}_{3} \mathrm{O}^{+}{ }_{\mathrm{IL}} \leftrightarrow \mathrm{DCH} 18 \mathrm{C} 6 \cdot \mathrm{Na}^{+}{ }_{\mathrm{IL}}+\mathrm{H}_{3} \mathrm{O}^{+}{ }_{\text {aq }}
\end{gathered}
$$

Here, increasing the DCH18C6 concentration promotes formation of additional hydronium ioncrown ether complex, which can then undergo ion exchange (IX) with the sodium ion present.

For strontium extraction into the $\mathrm{IL}$, the values of $\mathrm{D}_{\mathrm{M}}$ eventually begin to decline (albeit slightly) as the extractant concentration rises. This decline becomes even more evident as the aqueous acidity is decreased (Figure 7). These observations can be explained by recognizing that under the experimental conditions, a not insignificant contribution to the overall extraction of strontium is made by so-called "crown ether-mediated ion exchange" $(50,51)$, analogous to the process shown for sodium ion extraction above. In this instance, however, the initial formation of the 1:1 hydronium ion-crown ether complex is followed by a process in which two molecules of this adduct react with the metal ion, as necessitated by the electroneutrality requirement:

$$
\mathrm{Sr}^{2+}{ }_{\mathrm{aq}}+2 \mathrm{DCH} 18 \mathrm{C} 6 \cdot \mathrm{H}_{3} \mathrm{O}_{\mathrm{IL}}^{+} \leftrightarrow \mathrm{DCH} 18 \mathrm{C} 6 \cdot \mathrm{Sr}^{2+}{ }_{\mathrm{IL}}+2 \mathrm{H}_{3} \mathrm{O}_{\mathrm{aq}}^{+}+\mathrm{DCH} 18 \mathrm{C} 66_{\mathrm{IL}}
$$

Clearly a process such as this, which results in the production of free DCH18C6 molecules, would not be expected to be favored by increasing concentrations of extractant in the IL phase. Thus, as the initial concentration of the free crown ether in the IL phase is increased, the propensity to extract a divalent cation by crown ether-mediated IX is likely to diminish. The 
result, apparently, can be a decline in $\mathrm{D}_{\mathrm{M}}$ with increasing extractant concentration. Such an observation is, to the best of our knowledge, without precedent.

Sorption capacity studies. An obvious solution to the problem of extractant precipitation is to reduce its concentration in the IL. Such a reduction, however, would also lower the strontium sorption capacity relative to that of the commercial resin. If, for example, the concentration of DtBuCH18C6 in the IL were reduced to its solubility limit ( $c a .0 .6 \mathrm{M})$, a $21 \%$ decline in the capacity vs. that of a 1-octanol-based sorbent (from 4.74 to $3.74 \mathrm{mg} / \mathrm{mL}$ of bed) would be expected. Capacity determinations on the $\mathrm{C}_{10} \mathrm{C}_{1} \mathrm{imTf}_{2} \mathrm{~N}$-based and conventional sorbents, however, yield an even greater difference between the actual capacities of the two materials. That is, while the measured capacity of the commercial resin is (in agreement with a prior report (6)) $74 \%$ of the theoretical value $(3.51 \mathrm{mg} / \mathrm{mL})$, that of the IL-based resin is only $1.78 \mathrm{mg} / \mathrm{mL}, 57 \%$ of its theoretical value. Given that precipitation of the crown ether is not expected at this concentration, lower accessibility of the pore volume of the sorbent due to the high stationary phase viscosity is a likely contributor to the lower than anticipated capacity.

Sol-gel encapsulated crown ether-ionic liquid mixtures. That the properties of the support can exert an influence on the behavior of an extraction chromatographic material is by now well established (62). In 1977, for example, Parrish (62) showed that the rate of copper (II) uptake by the extractant Kelex 100 supported on any of a variety of macroporous polymers (e.g., XAD-series resins) was affected by the water regain (i.e., hydrophobicity) of the support, with more hydrophilic materials yielding faster copper ion uptake under a given set of conditions. Subsequent work by other investigators $(42,63-67)$ has confirmed the often significant influence of support characteristics on the chromatographic performance of EXC resins. Such results suggest that it may be possible to at least partly overcome the apparent limitations of the polymer-supported crown ether-IL system by appropriate choice of support. Recent work by Makote and Dai (34) concerning the properties of ionic liquid-crown ether mixtures encapsulated in sol-gel glasses lends additional credence to this notion. In particular, these investigators demonstrated that while a DCH18C6-impregnated sol-gel glass exhibited only limited uptake of strontium $\left(\mathrm{D}_{\mathrm{w}}=0.5\right)$ from a $\mathrm{pH} 4$ aqueous phase when undiluted crown ether was employed, addition of the ionic liquid 1-ethyl-3-methylimidazolium bis[(trifluoromethyl)sulfonyl]imide $\left(\mathrm{C}_{2} \mathrm{C}_{1} \mathrm{imTf}_{2} \mathrm{~N}\right)$ resulted in a dramatic (i.e., thousand-fold) increase in strontium retention under 
the same conditions. This suggests that a sol-gel glass matrix may be especially well suited as the basis for metal ion sorbents employing crown ether-ionic liquid mixtures.

With this in mind, sol-gel glasses (Figure 8) incorporating either undiluted DtBuCH18C6 or its solution $(1 \mathrm{M})$ in $\mathrm{C}_{10} \mathrm{C}_{1} \mathrm{imTf}_{2} \mathrm{~N}$ were prepared and characterized. For purposes of comparison, a third material incorporating a $1 \mathrm{M}$ solution of the crown ether in 1-octanol was also examined. Figure 9 depicts the nitric acid dependence of the weight distribution ratio, $\mathrm{D}_{\mathrm{w}}$, for strontium by these sorbents. As shown, in contrast to the results obtained by Makote (34) for DCH18C6, the sorbent incorporating only DtBuCH18C6 displays significant retention of radiostrontium, yielding a $\mathrm{D}_{\mathrm{w}}$ of nearly 80 at the highest acidities examined. Similar strontium ion retention behavior is seen for the sorbent incorporating a solution of DtBuCH18C6 in 1octanol, although a decline in the acid dependency is seen at sufficiently high aqueous acidities. This observation is consistent with previous results for polymer-based EXC resins, for which little effect on strontium ion retention by a crown ether-loaded support was noted upon introduction of 1-octanol (4). Again in contrast to the results of Makote (34), however, much the same strontium ion uptake is observed for the sorbent incorporating a solution of the crown ether in the ionic liquid. Apparently then (for reasons which remain unclear at present), the effect of the presence of an ionic liquid on the metal ion sorption behavior of a crown ether-loaded sol-gel glass is dependent upon the crown compound. A comparison of these data to those shown in Figure 4, in which the sorbents consist of solutions of the same extractant in 1-octanol or $\mathrm{C}_{10} \mathrm{C}_{1} \mathrm{imTf}_{2} \mathrm{~N}$ supported on Amberlite XAD-7, unexpectedly indicate that better results (as reflected in the magnitude of $D_{w}$ for strontium ion at a given acidity) are generally obtained for the polymer-based materials.

A further indication of the superiority of XAD-based sorbents is seen in measurements of the kinetics of strontium ion uptake. Figure 10 shows the results of these measurements for a sol-gel glass incorporating either undiluted DtBuCH18C6 or its solution (1 M) in 1-octanol or $\mathrm{C}_{10} \mathrm{C}_{1} \mathrm{imTf}_{2} \mathrm{~N}$. For the sol-gel-based materials, equilibration times exceeding two hours are observed, consistent with prior reports for sol-gel glasses incorporating a tetracarboxylic acidfunctionalized aza-crown ether (70) or a thiacrown ether (71), for which equilibration times of up to 24 hours have been found. For the XAD-based sorbents, however, published reports indicate that strontium uptake for both the "solvent-less" (4) and conventional $(5,6)$ resin is typically 
complete in 40 minutes or less, with the former exhibiting slightly slower uptake, a result consistent with the higher viscosity of the undiluted extractant.

\section{Conclusions}

The results presented here, while preliminary, do indicate that the transfer to a solidsupported (i.e., extraction chromatographic) configuration of metal ion separations methodology developed using ionic liquid-based liquid-liquid extraction systems may not be straightforward. Most notably, the significant stationary phase viscosities arising from the high extractant concentrations required to maximize the uptake of the ions of interest and the inherently high viscosity of typical ILs reduce the efficiency of IL-based EXC materials. Along these same lines, given the microheterogeneous nature of ILs incorporating long alkyl chains (59-61), in particular, the existence therein of alkane-like regions, and the need for such hydrophobic ILs to suppress undesirable extraction pathways (i.e., ion-exchange processes (14)), along with the modest solubility of various extractants (and/or extracted complexes) in unmodified alkanes (68, 69), inadequate extractant solubility in the IL phase represents another significant potential problem area.

This is not to say that ILs cannot provide the basis of EXC materials. Indeed, as already noted, a number of sorbents have already been described in which an IL alone is employed as both the diluent and the extractant. Much work remains to be done, however, before sorbents incorporating extractant-IL combinations can achieve their full potential. Among the variety of unresolved issues, the optimum level of extractant-diluent loading on the support, the preferred support hydrophobicity and porosity, and the characteristics of the extractant and the IL yielding the most satisfactory stationary phase behavior are especially important. Work to address these issues is now underway in our laboratories. 


\section{Acknowledgements}

The authors gratefully acknowledge the financial support of this work by the Office of Basic Energy Sciences of the United States Department of Energy under the Single Investigator Small Group Research (SISGR) Program through sub-contract with Brookhaven National Laboratory (liquid-liquid extraction studies); the PG Research Foundation (isolation of single isomeric forms of DtBuCH18C6); and the Centers for Disease Control and Prevention, National Center for Environmental Health, Division of Laboratory Sciences (sorbent characterization). 


\section{References}

1. M.L. Dietz, E.P. Horwitz, Novel chromatographic materials based on nuclear waste processing chemistry, LC-GC, 11 (1993) 424-436.

2. M.L. Dietz, E.P. Horwitz, A.H. Bond, Extraction chromatography: Progress and opportunities, in: A.H. Bond, M.L. Dietz, R.D. Rogers, (Eds.) Metal ion separation and preconcentration: Progress and opportunities, American Chemical Society, Washington, DC, (1999) 234-250.

3. M.L. Dietz, Recent progress in the development of extraction chromatographic methods for radionuclide separation and preconcentration, in: C.A. Laue, K. L. Nash, (Eds.), Radioanalytical methods in interdisciplinary research: Fundamentals in cutting edge applications, American Chemical Society, Washington, DC, (2004) 161-176.

4. Dietz, M.L.; Yaeger, J.; Sajdak, L.R.; Jensen, M.P. Characterization of an improved extraction chromatographic material for the separation and preconcentration of strontium from acidic media, Sep. Sci. Technol. 40 (2005) 349-366.

5. E.P. Horwitz, M.L. Dietz, D.E. Fisher, Separation and preconcentration of strontium from biological, environmental, and nuclear waste samples by extraction chromatography using a crown ether, Anal. Chem. 63 (1991) 522-525.

6. E.P. Horwitz, R. Chiarizia, M.L. Dietz, A novel strontium-selective extraction chromatographic resin, Solv. Extr. Ion Exch. 10 (1992) 313-336.

7. R. Chiarizia, E.P. Horwitz, M.L. Dietz, Acid dependency of the extraction of selected metal ions by a strontium-selective extraction chromatographic resin: Calculated vs. experimental curves, Solv. Extr. Ion Exch. 10 (1992) 337-361.

8. E.P. Horwitz, M.L. Dietz, D.E. Fisher, Correlation of the extraction of strontium nitrate by a crown ether with the water content of the organic phase, Solv.Ext. Ion Exch. 8 (1990) 199-208.

9. M.L. Dietz, E.P. Horwitz, S. Rhoads, R. A. Bartsch, J. Krzykawski, Extraction of cesium from acidic nitrate media using macrocyclic polyethers, The role of organic phase water. Solv. Extr. Ion Exch. 14 (1996) 1-12.

10. E.P. Horwitz, M.L. Dietz, S. Rhoads, C. Felinto, N.H. Gale, J. Houghton, A leadselective extraction chromatographic resin and its application to the isolation of lead from geological samples, Anal. Chim. Acta 292 (1994) 263-273.

11. P. Bonhote, A.P. Dias, N. Papageorgiou, K. Kalyanasundaram, M. Grätzel, Hydrophobic, highly conductive ambient-temperature molten salts, Inorg. Chem. 35 (1996) 1168-1178.

12. S. Dai, Y.H. Ju, C.E. Barnes, Solvent extraction of strontium nitrate by a crown ether using room-temperature ionic liquids, J. Chem. Soc. Dalton Trans. (1999) 1201-1202. 
13. M.L. Dietz, J.A. Dzielawa, Ion-exchange as a mode of cation transfer into roomtemperature ionic liquids containing crown ethers: Implications for the "greenness" of ionic liquids as diluents in liquid-liquid extraction, Chem. Commun. (2001) 2124-2125.

14. M.L. Dietz, J.A. Dzielawa, I. Laszak, B.A. Young, M.P. Jensen, Influence of solvent structural variations on the mechanism of facilitated ion transfer into room-temperature ionic liquids, Green Chem. 5 (2003) 682-685.

15. K. Campos, R. Domingo, T. Vincent, M. Ruiz, A.M. Sastre, E. Guibal, Bismuth recovery from acidic solutions using Cyphos IL-101 immobilized in a composite biopolymer matrix, Water Res. 42 (2008) 4019-4031.

16. T. Vincent, A. Parodi, E. Guibal, Pt recovery using Cyphos IL-101 immobilized in biopolymer capsules, Sep. Purif. Technol. 62 (2008) 470-479.

17. E. Guibal, K.C. Gavilan, P. Bunio, T. Vincent, A. Trochimczuk, Cyphos IL-101 (tetradecyl(trihexyl)phosphonium chloride) immobilized in biopolymer capsules for $\mathrm{Hg}(\mathrm{II})$ recovery from HCl solutions, Sep. Sci. Technol. 43 (2008) 2406-2433.

18. V. Gallardo, R. Navarro, I. Saucedo, M. Avila, E. Guibal, Zinc(II) extraction from hydrochloric acid solutions using Amberlite XAD-7 impregnated with Cyphos IL 101 (tetradecyl(trihexyl)phosphonium chloride), Sep. Sci. Technol. 43 (2008) 2434-2459.

19. E. Guibal, T. Vincent, C. Jouannin, Immobilization of extractants in biopolymer capsules for the synthesis of new resins: a focus on the encapsulation of tetraalkylphosphonium ionic liquids, J. Mater. Chem. 19 (2009) 8515-8527.

20. X. Sun, B. Peng, Y. Ji, J. Chen, D. Li, Chitosan (chitin)/cellulose composite biosorbents prepared using ionic liquid for heavy metal ions adsorption, AIChE J. 55 (2009) 20622069.

21. S. Xiaoqi, J. Yang, C. Ji, M.A. Juitong, Solvent impregnated resin prepared using taskspecific ionic liquids for rare earth separation, J. Rare Earths 27 (2009) 932-936.

22. E. Guibal, A.F. Piñol, M. Ruiz, T. Vincent, C. Jouannin, A.M. Sastre, Immobilization of Cyphos ionic liquids in alginate capsules for Cd(II) sorption, Sep. Sci. Technol. 45 (2010) 1935-1949.

23. R. Navarro, I. Saucedo, M.A. Lira, E. Guibal, Gold(III) recovery from HCl solutions using Amberlite XAD-7 impregnated with an ionic liquid (Cyphos IL-101), Sep. Sci. Technol. 45 (2010) 1950-1962.

24. S. Ayata, S.S. Bozkurt, K. Ocakoglu, Separation and preconcentration of $\mathrm{Pb}(\mathrm{II})$ using ionic liquid-modified silica and its determination by flame atomic absorption spectrometry, Talanta 84 (2011) 212-215. 
25. R. Navarro, I. Saucedo, C. Gonzalez, E. Guibal, Amberlite XAD-7 impregnated with Cyphos IL-101 (tetraalkylphosphonium ionic liquid) for $\mathrm{Pd}(\mathrm{II})$ recovery from $\mathrm{HCl}$ solutions, Chem. Eng. J. 185-186 (2012) 226-235.

26. Y. Liu, L. Zhu, X. Sun, J. Chen, F. Luo, Silica materials doped with bifunctional ionic liquid extractant for yttrium extraction, Ind. Eng. Chem. Res. 48 (2009) 7308-7313.

27. Y. Liu, X. Sun, F. Luo, J. Chen, The preparation of sol-gel materials doped with ionic liquids and trialkyl phosphine oxides for yttrium(III) uptake, Anal. Chim. Acta 604 (2007) 107-113.

28. G.V. Myasoedova, E.A. Zakharchenko, N.P. Molochnikova, B.F. Myasoedov, Solid extractants prepared with ionic liquids and their use for recovery of actinides from nitric acid solutions, Radiochemistry 50 (2008) 482-485.

29. X. Sun, B. Peng, Y. Ji, J. Chen, D. Li, The solid-liquid extraction of yttrium from rare earths by solvent (ionic liquid) impregnated resin coupled with complexing method. Sep. Purif. Technol. 63 (2008) 61-68.

30. B. Yu, J.R. Bell, H. Luo, S. Dai, Ionic liquid and silica sol-gel composite materials doped with $N, N, N^{\prime}, N^{\prime}$-tetra( $n$-octyl)diglycolamide for extraction of $\mathrm{La}^{3+}$ and $\mathrm{Ba}^{2+}$, Sep. Sci. Technol. 47 (2012) 244-249.

31. E.P. Horwitz, M.L. Dietz, D.M. Nelson, J.J. LaRosa, W.D. Fairman, Concentration and separation of actinides from urine using a supported bifunctional organophosphorus extractant, Anal. Chim. Acta 238 (1989) 263-271.

32. M. Deetlefs, K.R. Seddon, Improved preparations of ionic liquids using microwave irradiation, Green Chem. 5 (2003) 181-186.

33. C.A. Hawkins, A. Rud, S.L. Garvey, M.L. Dietz, Evaluation of hydroxyalkylfunctionalized imidazolium-based ionic liquids as solvents for the extraction of metal ions, Sep. Sci.Technol. 47 (2012) 1993-2001.

34. R.D. Makote, H. Luo, S. Dai, Synthesis of ionic liquid and silica composites doped with dicyclohexyl-18-crown-6 for sequestration of metal ions, in: M.A. Abraham, L. Moens (Eds.) Clean Solvents: Alternative Media for Chemical Reactions and Processing, American Chemical Society, Washington, DC, (2002) 26-33.

35. S. Dai, S.Y.H. Ju, H.J. Gao, J.S. Lin, S.J. Pennycook, C.E. Barnes, Preparation of silica aerogel using ionic liquids as solvents, Chem. Commun. (2000) 243-244.

36. E.P. Horwitz, D.R. McAlister, M.L. Dietz, Extraction chromatography versus solvent extraction: How similar are they?, Sep. Sci. Technol. 41 (2006) 2163-2182.

37. T.B. Pierce, R.S. Hobbs, The separation of rare earths by partition chromatography with reversed phases. Part I. Behavior of column material, J. Chrom. 12 (1963) 74-80. 
38. T.B. Pierce, P.F. Peck, R.S. Hobbs, The separation of rare earths by partition chromatography with reversed phases. Part II. Behaviour of individual elements on HDEHP-corvic columns, J. Chrom. 12 (1963) 81-88.

39. I. Akaza, T. Kiba, T. Kiba, The separation of gold, platinum, and palladium by reversed phase partition chromatography, Bull. Chem. Soc. Jpn. 43 (1970) 2063-2067.

40. F. Sebesta, Extraction chromatography using chelating agents. I. System zinc-dithizonecarbon tetrachloride, J. Radioanal. Chem. 6 (1970) 41-46.

41. F. Sebesta, Extraction chromatography using chelating agents. II. Separation of some radioisotopes by dithizone, J. Radioanal. Chem. 7 (1971) 41-47.

42. K. Isshiki, F. Tsuji, T. Kuwamoto, E. Nakayama, Preconcentration of trace metals from seawater with 7-dodecenyl-8-quinolinol impregnated macroporous resin, Anal. Chem. 59 (1987) 2491-2495.

43. J.L. Cortina, N. Miralles, M. Aguilar, A.M. Sastre, Solvent impregnated resins containing di(2-ethylhexyl)phosphoric acid. II. Study of the distribution equilibria of $\mathrm{Zn}(\mathrm{II}), \mathrm{Cu}(\mathrm{II})$, and Cd(II), Solv. Extr. Ion Exch. 12 (1994) 371-391.

44. A.G. Strikovsky, K. Jerabek, J.L. Cortina, A.M. Sastre, A. Warshawsky, Solvent impregnated resin (SIR) containing dialkyldithiophosphoric acid on Amberlite XAD-2: Extraction of copper and comparison to liquid-liquid extraction, React. Funct. Polym. 28 (1996) 149-158.

45. R.M. Izatt, J.S. Bradshaw, S.A. Nielsen, J.D. Lamb, J.J. Christensen, Thermodynamic and kinetic data for cation macrocycle interaction, Chem. Rev. 85 (1985) 271-339.

46. R.M. Izatt, K. Pawlak, J.S. Bradshaw, R.L. Bruening, Thermodynamic and kinetic data for macrocycle interactions with cations and anions, Chem. Rev. 91 (1991) 1721-2085.

47. E.P. Horwitz, M.L. Dietz, D.E. Fisher, SREX: A New Process for the Extraction and Recovery of Strontium from Acidic Nuclear Waste Streams, Solvent Extr. Ion Exch. 9 (1991) 1-25.

48. E.P. Horwitz, M.L. Dietz, D.E. Fisher, Extraction of strontium from nitric acid solutions using dicyclohexano-18-crown-6 and its derivatives, Solv. Extr. Ion Exch. 8 (1990) 557572.

49. M.L. Dietz, A.H. Bond, M. Clapper, J.W. Finch, Isomer effects in the extraction of metal ions from acidic nitrate media by dicyclohexano-18-crown-6, Radiochim. Acta 85 (1999) 119-129. 
50. M.L. Dietz, D.C. Stepinski, A ternary mechanism for the facilitated transfer of metal ions into room-temperature ionic liquids (RTILs): implications for the "greenness" of RTILs as extraction solvents, Green Chem. 7 (2005) 747-750.

51. C.A. Hawkins, S.L. Garvey, M.L. Dietz, Structural variations in room-temperature ionic liquids: Influence on metal ion partitioning modes and extraction selectivity, Sep. Purif. Technol. 89 (2011) 31-38.

52. R.M. Izatt, B.L. Haymore, J.S. Bradshaw, Christensen, J.J. Facile separation of the cis isomers of dicyclohexano-18-crown-6, Inorg. Chem. 14 (1975) 3132-3133.

53. M.L. Dietz, C. Felinto, S. Rhoads, M. Clapper, J.W. Finch, B.P. Hay, Comparison of column chromatographic and precipitation methods for the purification of a macrocyclic polyether extractant, Sep. Sci. Technol. 34 (1999) 2943-2956.

54. A.J. Pawlak, Crown ether stereoisomerism: Implications in metal ion extraction and ionic liquid design, Ph.D. dissertation, University of Wisconsin-Milwaukee, 2014.

55. T. Braun, G. Ghersini, Extraction chromatography, Elsevier, New York, 1975.

56. C.C. Miller, The Stokes-Einstein Law for diffusion in solution, Proceedings of the Royal Society of London. Series A. Containing Papers of a Mathematical and Physical Character 106 (1924) 724-749.

57. S. Matsuo, T. Makita, Viscosities of six 1-alkanols at temperatures in the range 298-348 K and pressures up to 200 MPA, Int. J. Thermophys. 10 (1989) 833-843.

58. J.G. Huddleston, A.E. Visser, W.M. Reichert, G.A. Broker, R.D. Rogers, Characterization and comparison of hydrophilic and hydrophobic room temperature ionic liquids incorporating the imidazolium cation, Green Chem. 3 (2001) 156-164.

59. O. Russina, A. Triolo, L. Gontrani, R. Caminiti, D. Xiao, L.G. Hines Jr., R.A. Bartsch, E.L. Quitevis, N. Plechkova, K.R. Seddon, Morphology and intermolecular dynamics of 1-alkyl-3-methylimidazolium bis\{(trifluoromethane) sulfonyl $\}$ amide ionic liquids: structural and dynamic evidence of nanoscale segregation,. J. Phys.: Condens. Matter. 21 (2009) 424121.

60. A. Triolo, O. Russina, H.J. Bleif, E. DiCola, Nanoscale aggregation in room temperature ionic liquids, J. Phys. Chem. B 111 (2007) 4641-4644.

61. D. Xiao, J.R. Rajian, A. Cady, S. Li, R.A. Bartsch, E.L. Quitevis, Nanoscale organization and anion effects on the temperature dependence of the optical Kerr effect spectra of ionic liquids, J. Phys. Chem. B 111 (2007) 4669-4677.

62. J.R. Parrish, Macroporous resins as supports for a chelating liquid ion-exchanger in extraction chromatography, Anal. Chem. 49 (1977) 1189-1192. 
63. K. Isshiki, E. Nakayama, Selective concentration of cobalt in seawater by complexation with various ligands and sorption on macroporous resins, Anal. Chem. 59 (1987) 291295.

64. S. Blain, P. Appriou, H. Handel, Column preconcentration of trace metals from sea-water with macroporous resins impregnated with lipophilic tetraaza macrocycles, Analyst 116 (1991) 815-820.

65. R.-S. Juang, M.-L. Chen, Comparative equilibrium studies on the sorption of metal ions with macroporous resins containing a liquid ion-exchanger, Sep. Sci. Technol. 32 (1997) 1017-1035.

66. H. Matsunaga, A.A. Ismail, Y. Wakui, T. Yokoyama, Extraction of rare earth elements with 2-ethylhexyl hydrogen 2-ethylhexyl phosphonate impregnated resins having different morphologies and reagent content, React. Funct. Polym. 49 (2001) 189-195.

67. E.A. Bursali, M. Yurdakoç, M. Merdivan, The effect of degree of impregnation in Amberlite resins with organophosphorus extractants for Y(III), La(III), Ce(III), Th(IV) and U(VI) ions, Sep. Sci. Technol. 43 (2008) 1421-1433.

68. D.L. Kalina, E.P. Horwitz, Variations in the solvent extraction behavior of bifunctional phosphorus-based compounds modified with TBP, Solv. Extr. Ion Exch. 3 (1985) 235250.

69. M.L. Dietz, E.P. Horwitz, R.D. Rogers, Extraction of strontium from acidic nitrate media using a modified PUREX solvent, Solv. Extr. Ion Exch. 13 (1995) 1-17.

70. T.L. Yost, Jr., B.C. Fagan, L.R. Allain, C.E. Barnes, S. Dai, M.J. Sepaniak, Z. Xue, Crown ether-doped sol-gel materials for strontium separation, Anal. Chem. 72 (2000) 5516-5519.

71. B. Saad, C.C. Chong, A.S.M. Ali, M.F. Bari, I.A. Rahman, N. Mohamad, M.I. Saleh, Selective removal of heavy metal ions using sol-gel immobilized and SPE-coated thiacrown ethers, Anal. Chim. Acta 555 (2006) 146-156. 
Table I. Effect of DtBuCH18C6 concentration in 1-octanol or $\mathrm{C}_{10} \mathrm{C}_{1} \mathrm{imTf}_{2} \mathrm{~N}$ on the extraction of strontium ion from 1.0 M nitric acid solution

1-octanol

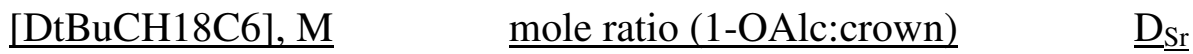

0.25

22

8.7

0.50

9.4

15.1

0.75

5.4

19.4

1.00

3.1

26.5

$\mathrm{C}_{10} \mathrm{C}_{1} i m T f_{2} \mathrm{~N}$

[DtBuCH18C6], M

$\underline{\text { mole ratio (IL:crown) }}$

$\underline{\mathrm{D}} \underline{\mathrm{r}}$

0.25

8.0

25.1

0.40

34.5

0.50

3.5

43.2

0.60

47.9

0.75

1.00

1.2

33.1

- a

${ }^{\text {a }}$ Precipitation of the crown ether is observed. 


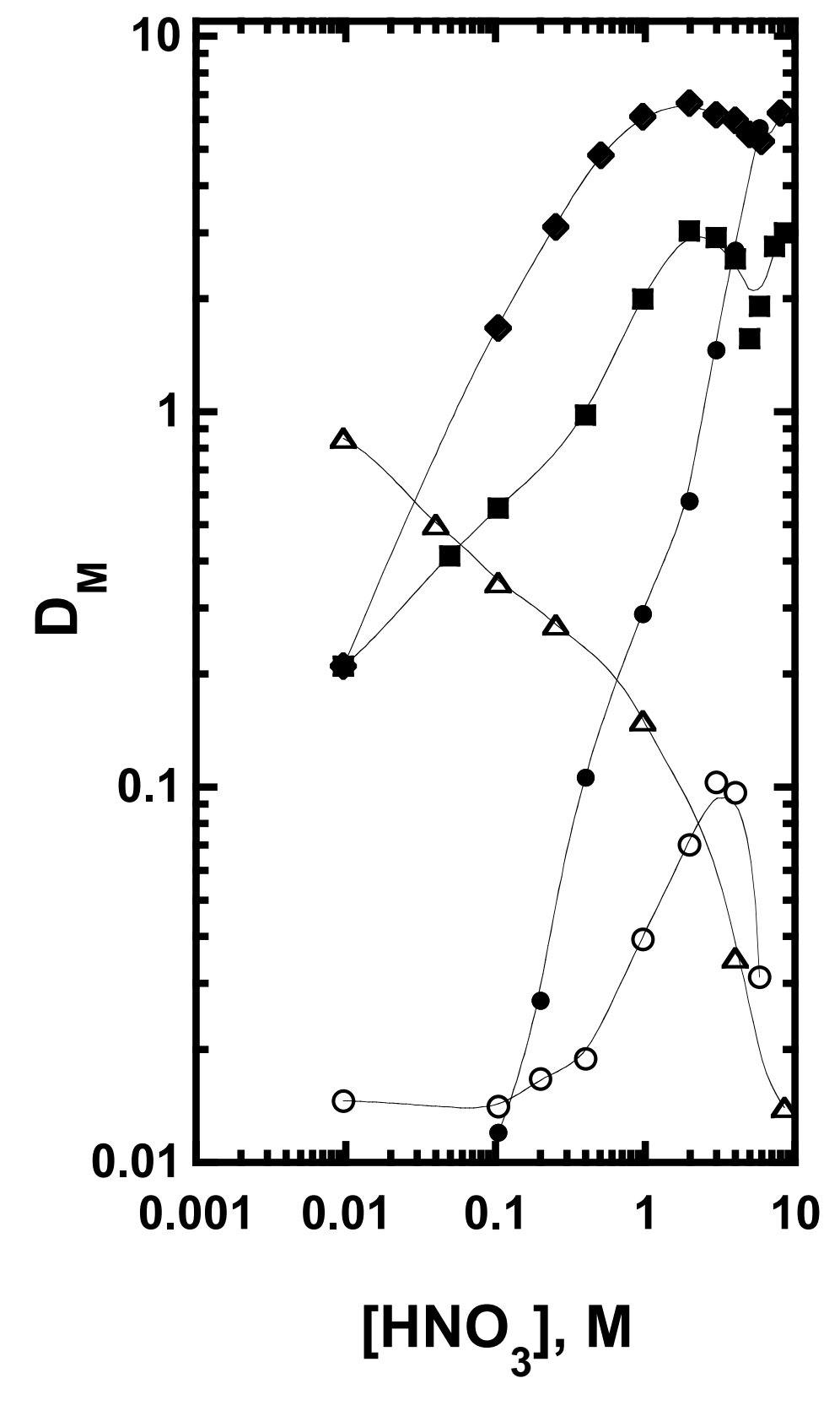

Figure 1.

Figure 


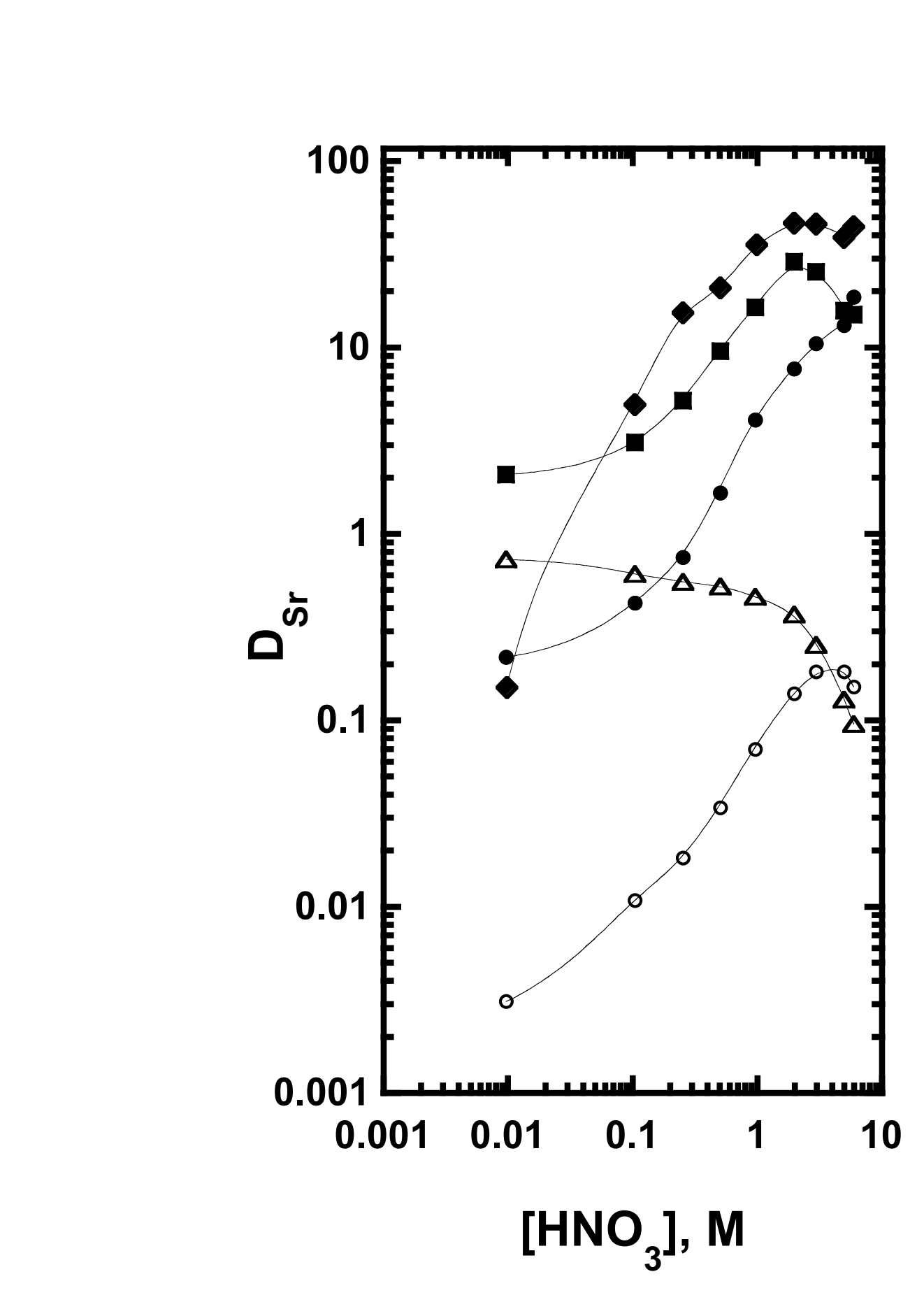

\author{
Figure 3. \\ Figure 3.
}
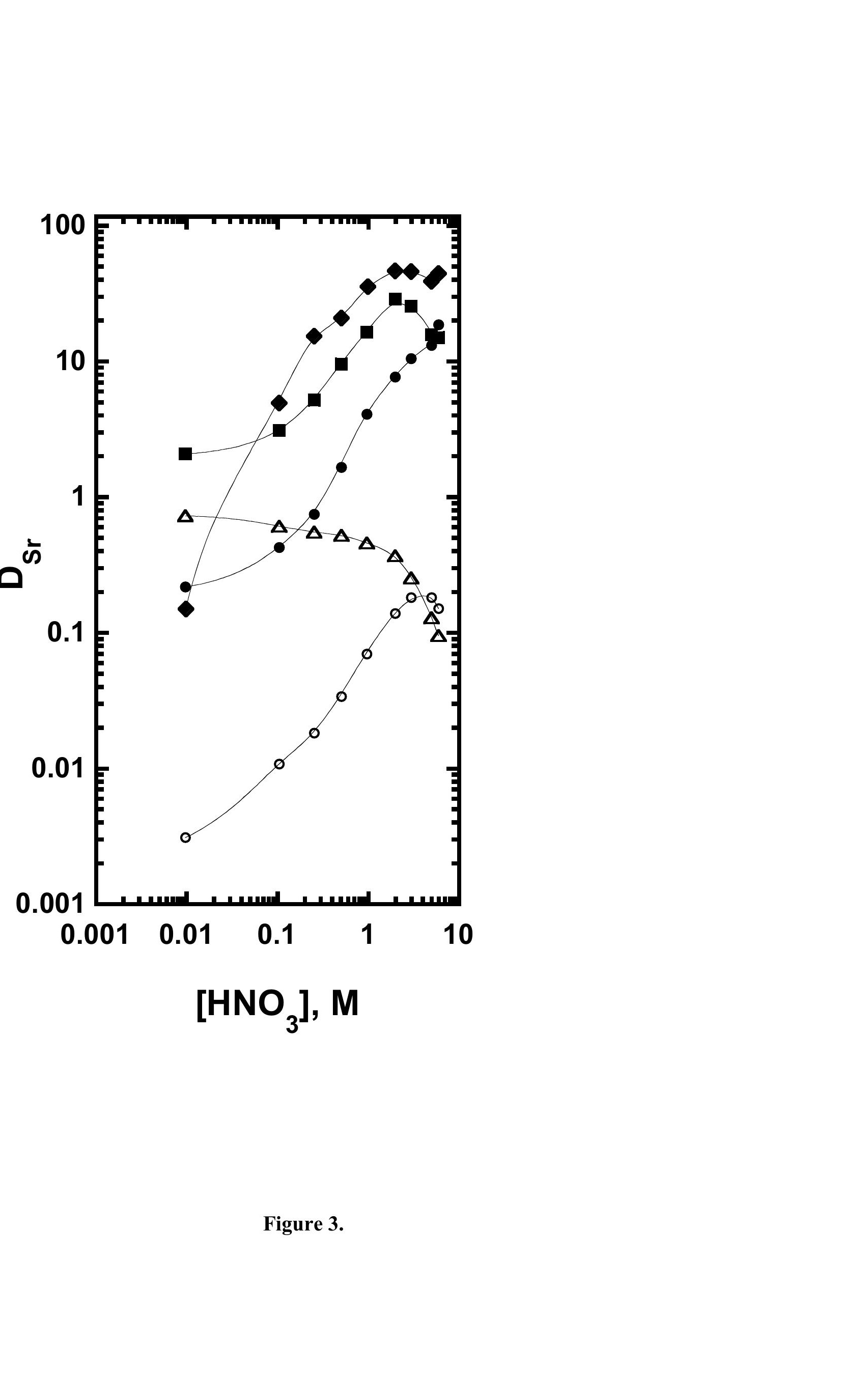


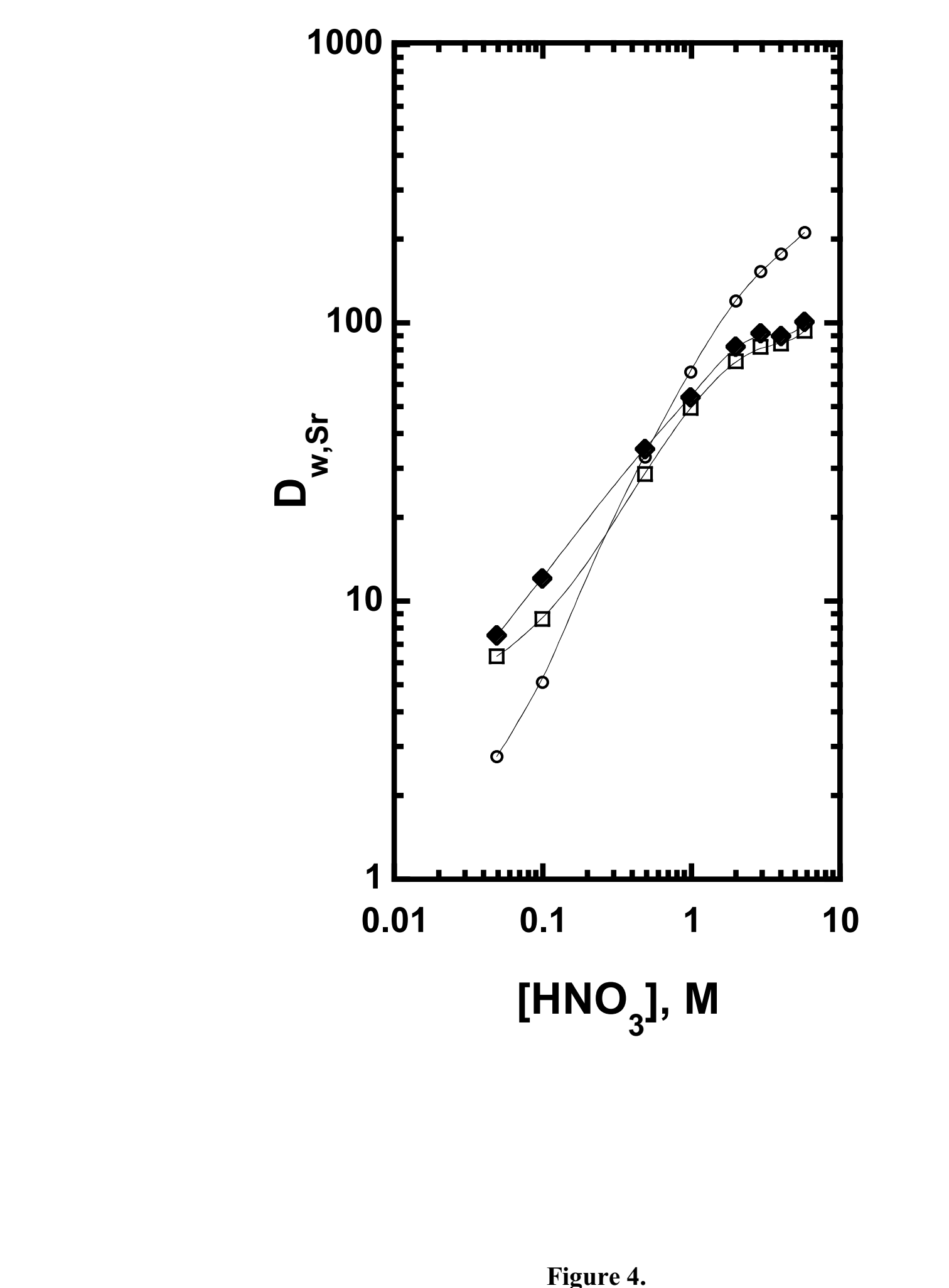

Figure 4.

Figure

Figure 4.

.

-

(n)

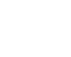

(1)

(1)

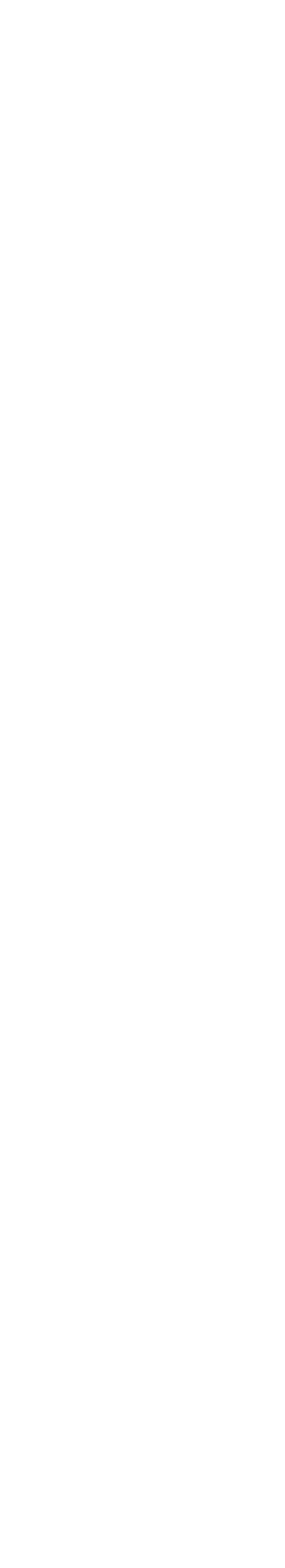

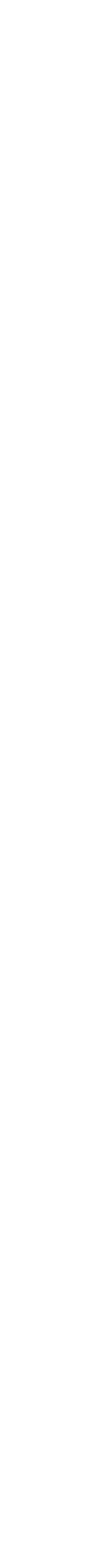
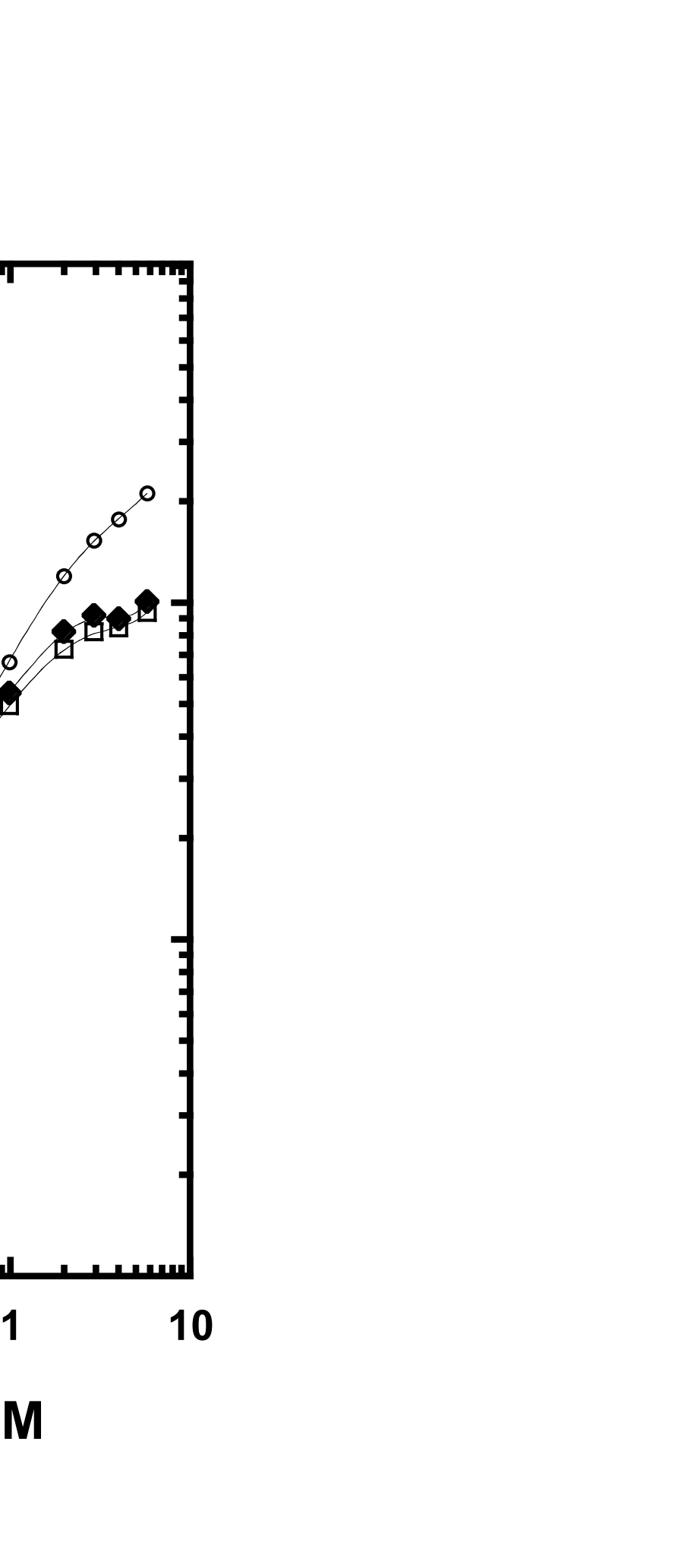

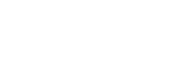

s. 


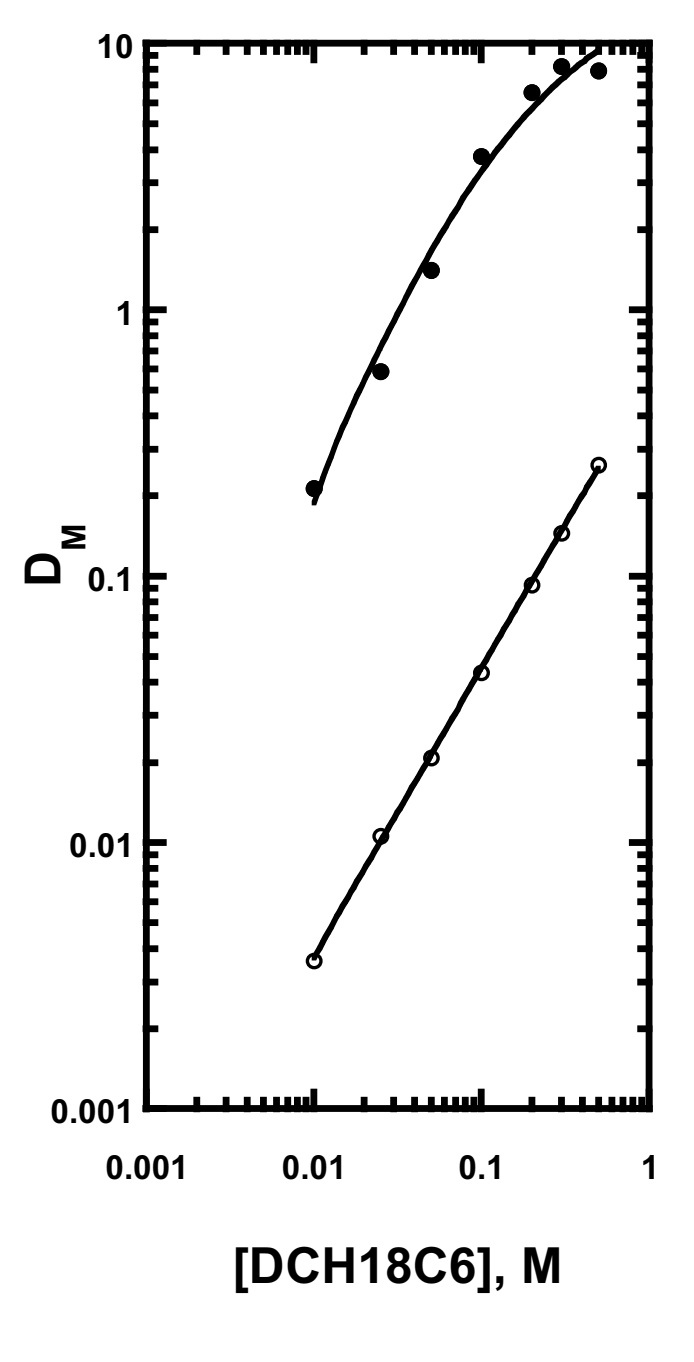

Figure 5A.

Figure

Figure 5A.

.

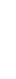

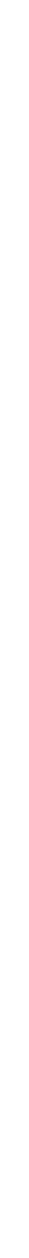




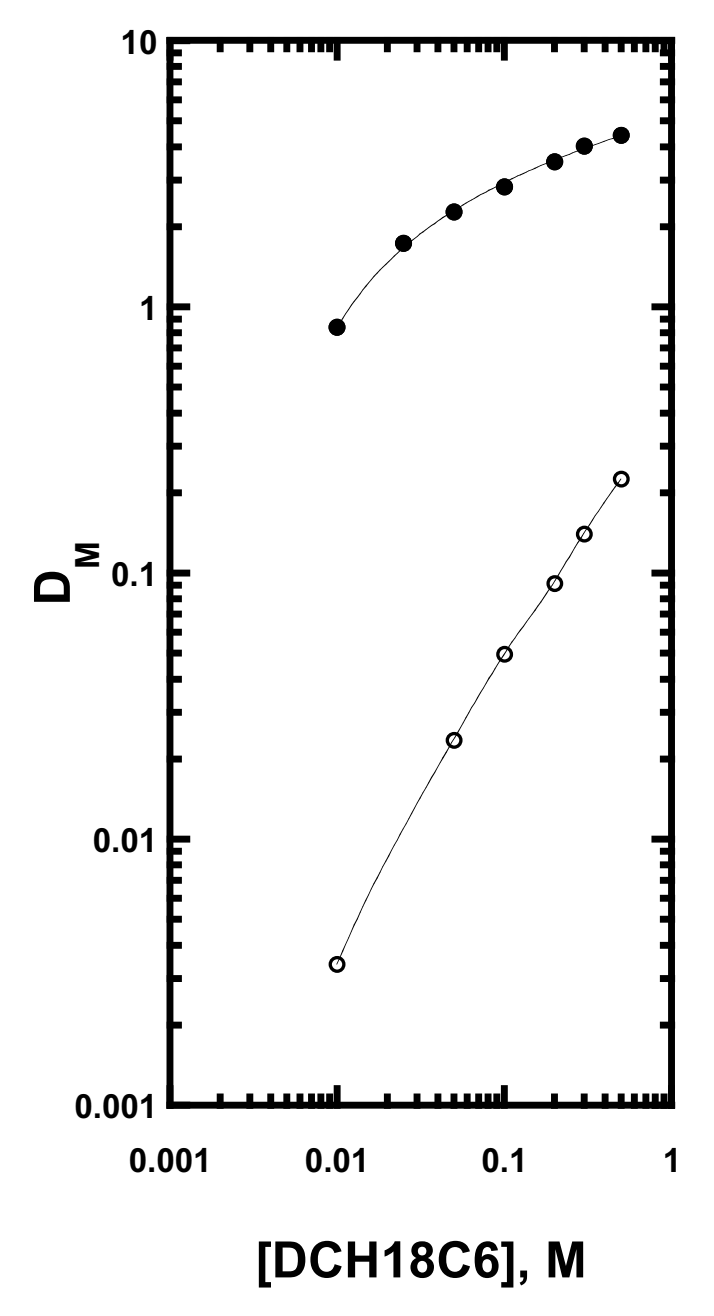

Figure 5B.

Figure

Figure 5B.

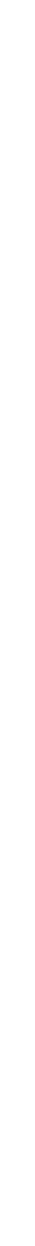

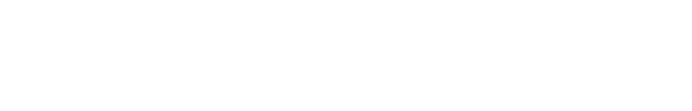

.
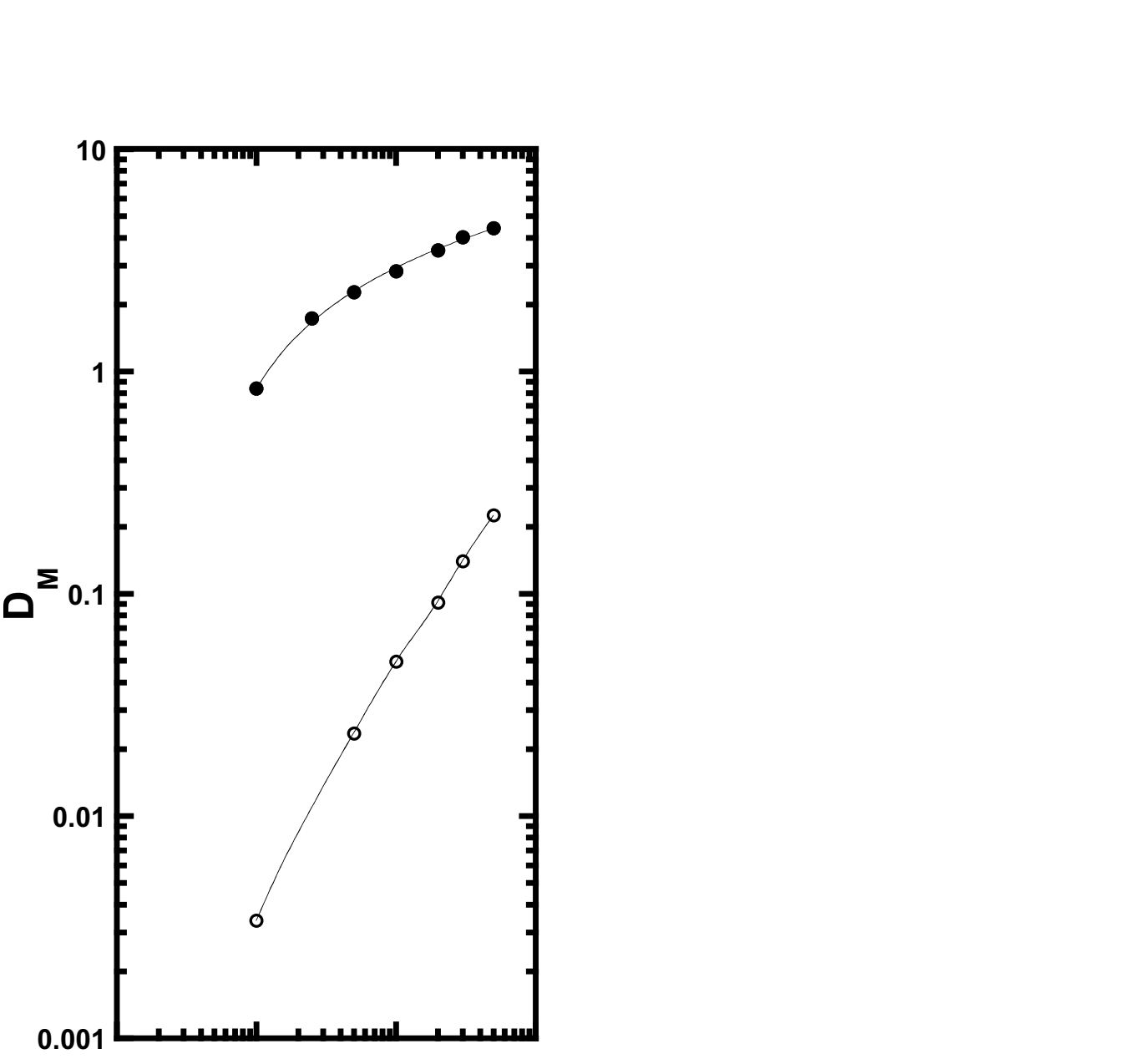

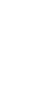

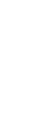

(

(
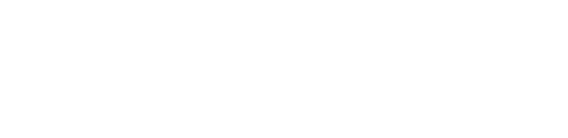

[DCH18C6], M 


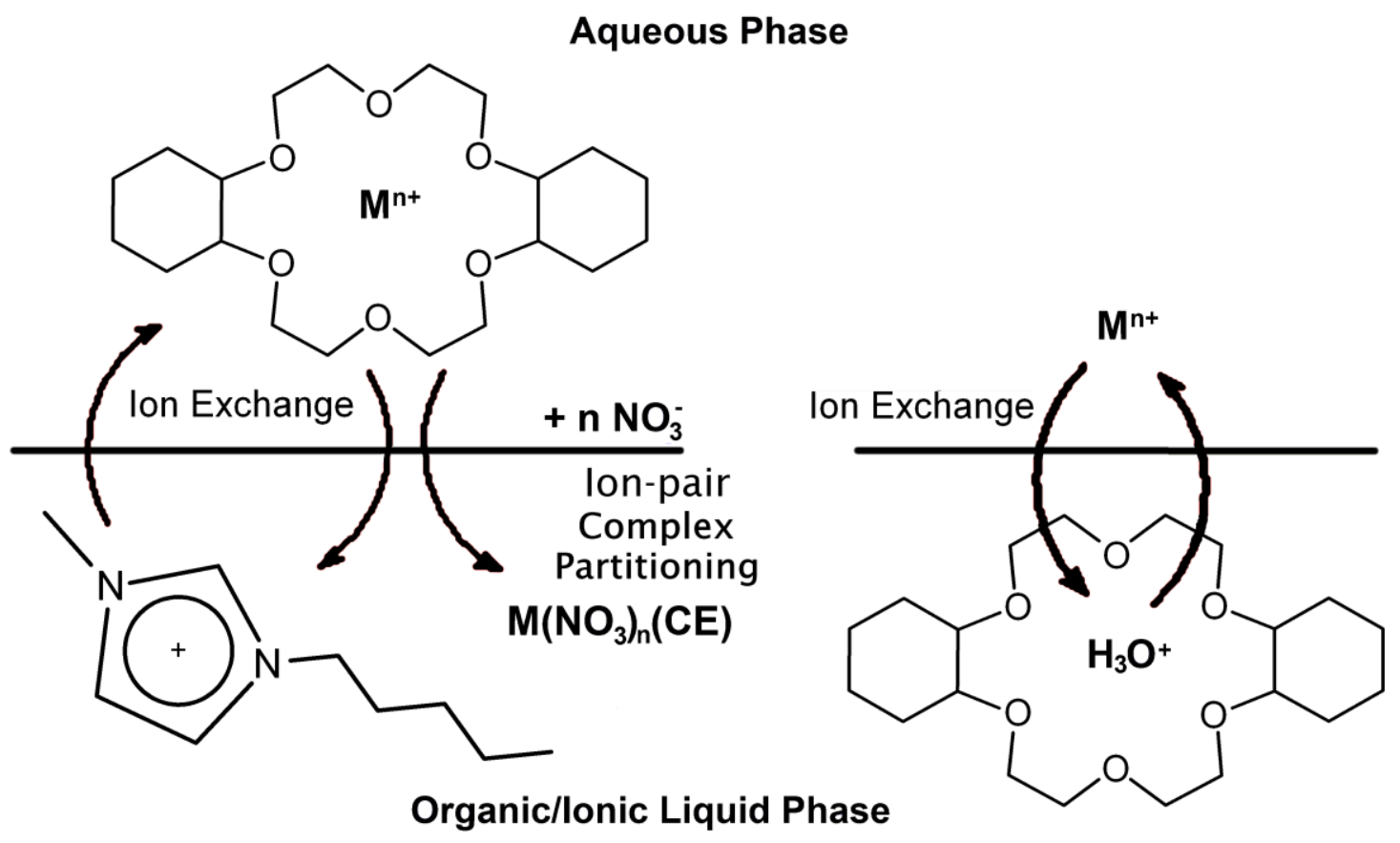

Figure 6. 


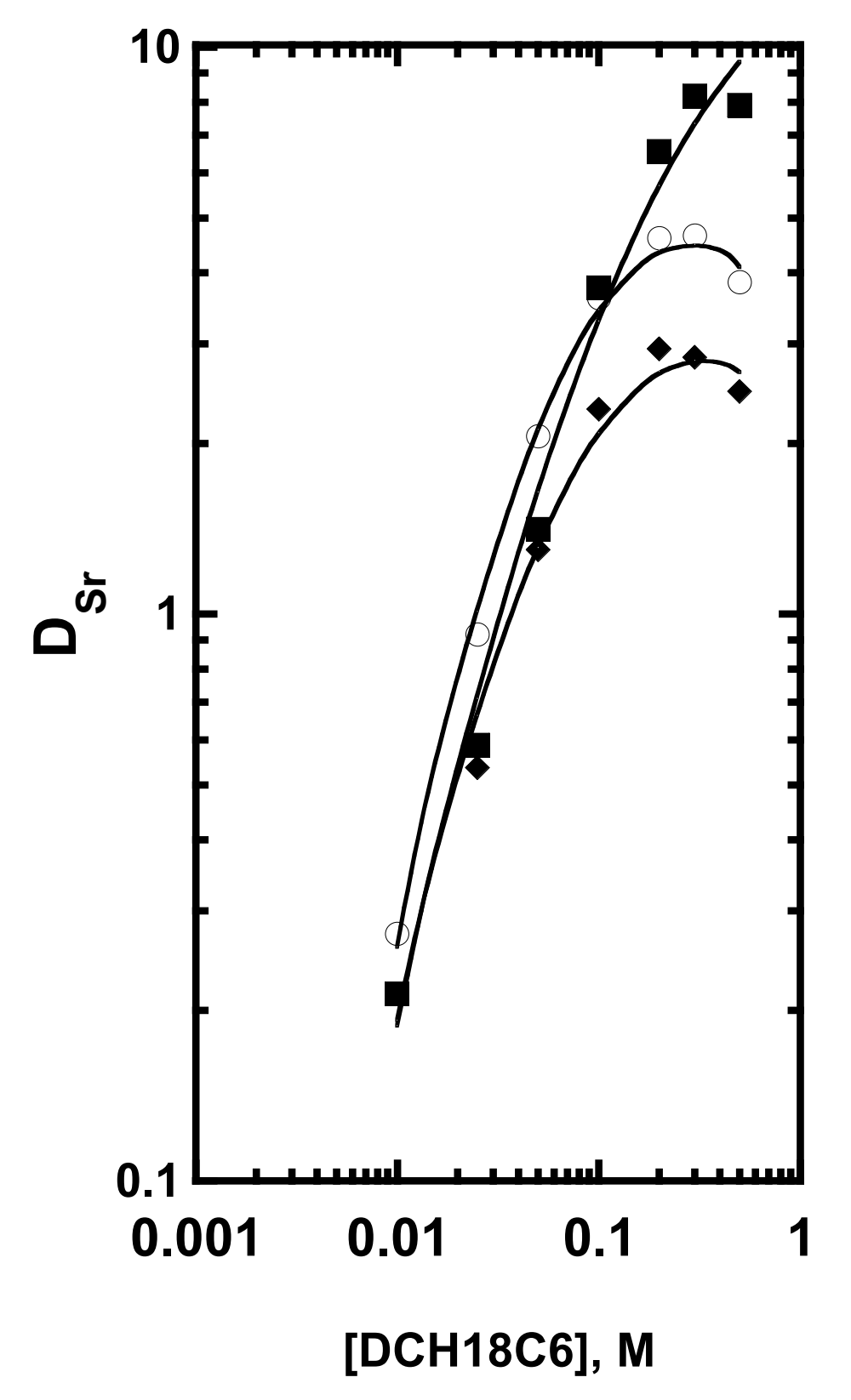

Figure 7.

Figure

Figur

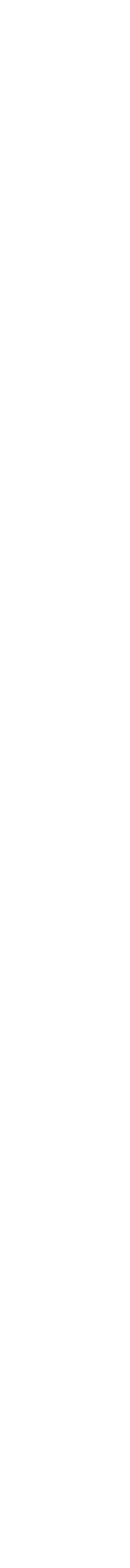

Figure 7.

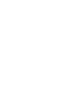$$
\text { . }
$$
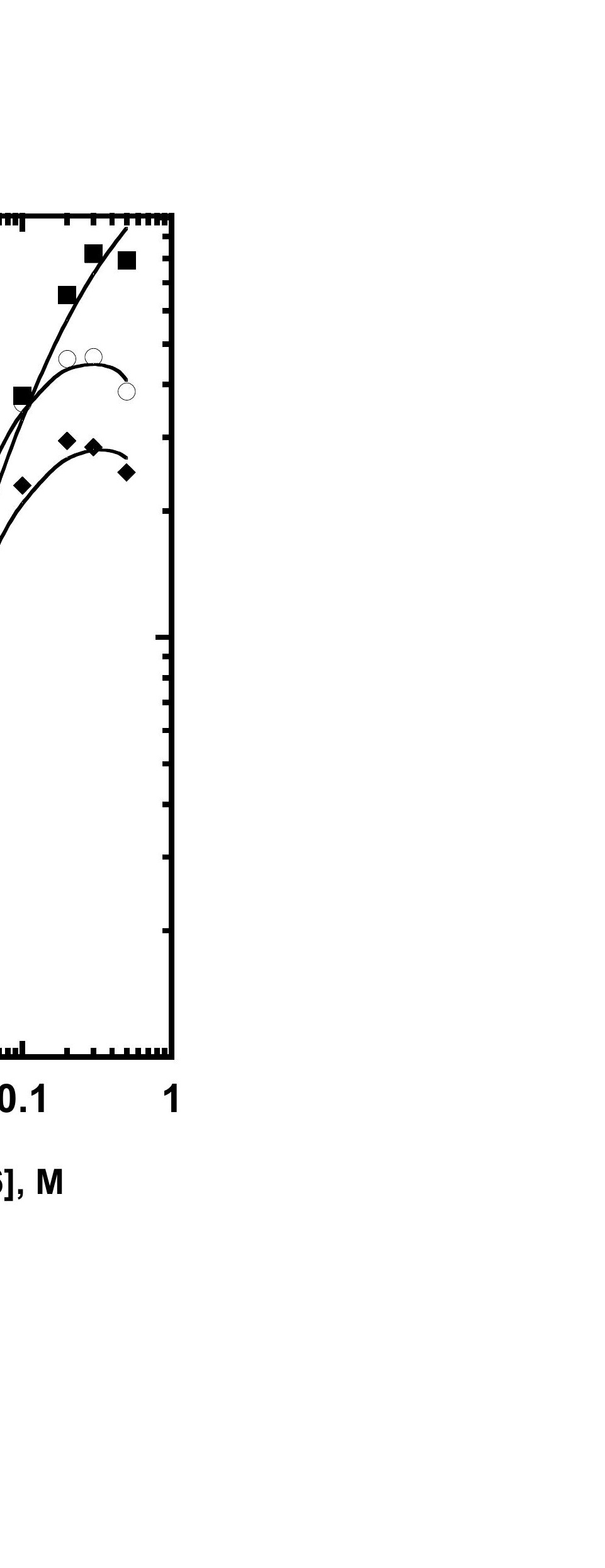

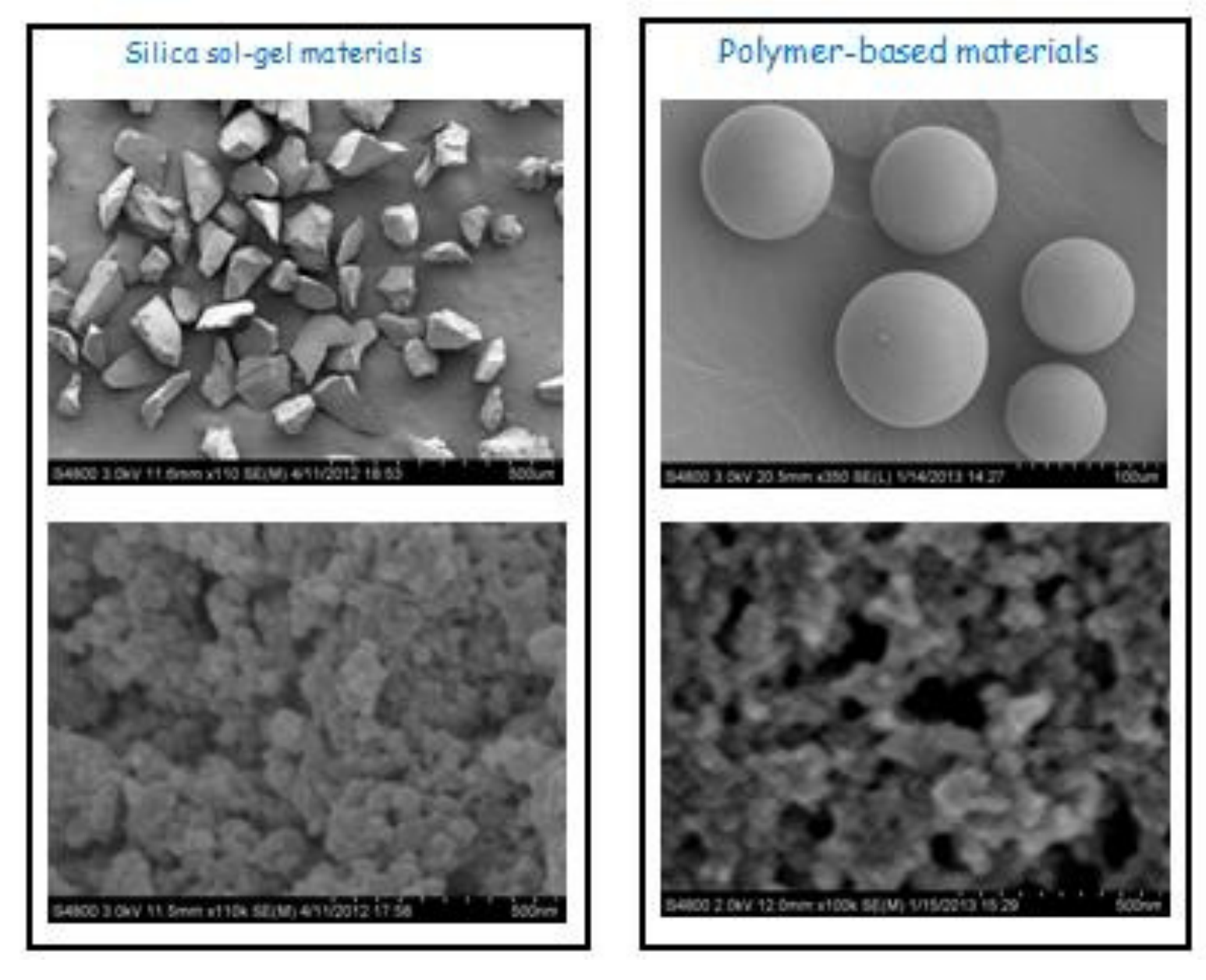

Figure 8. 


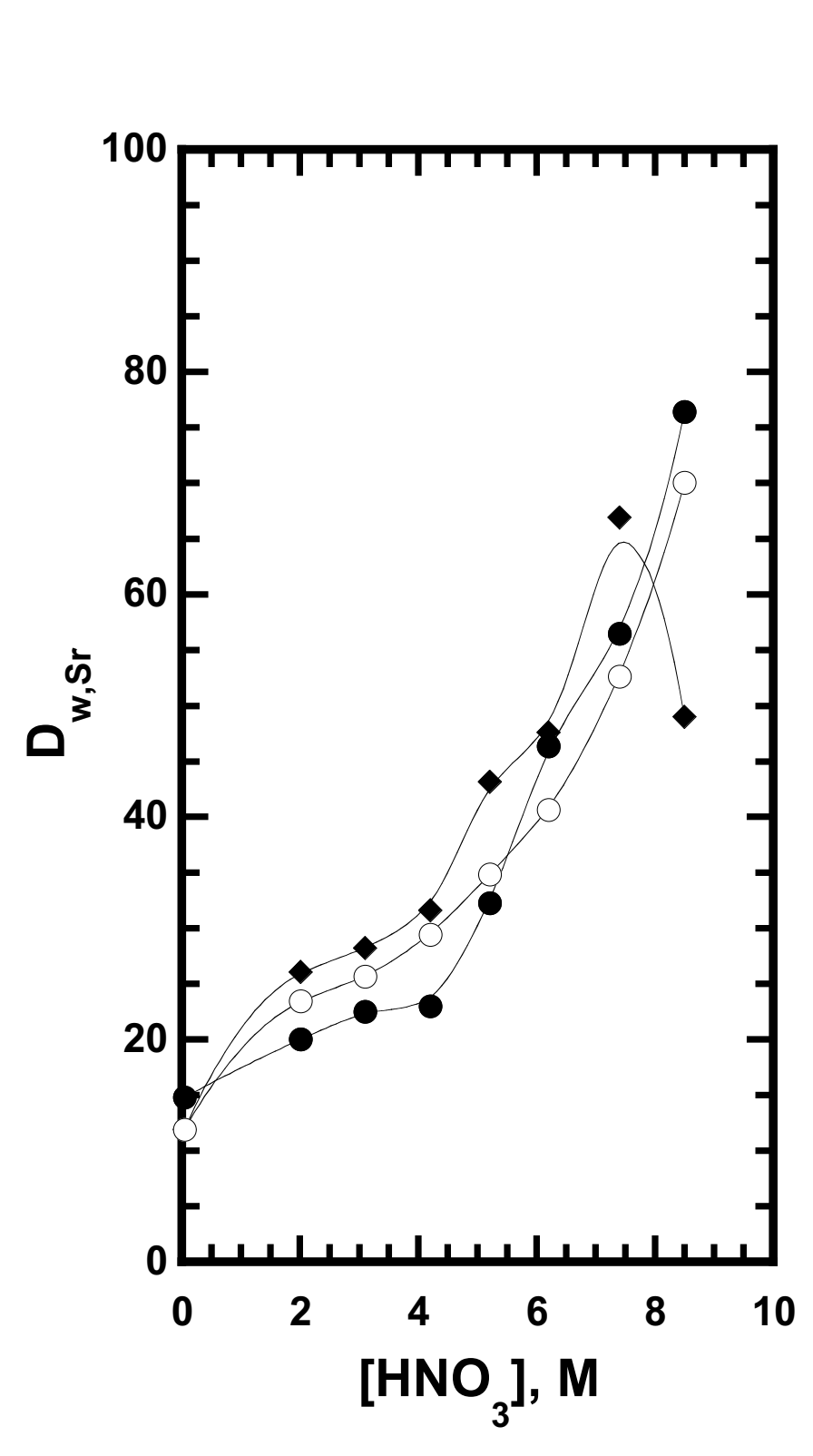

Figure 9.

\author{
Pigure 9.
}




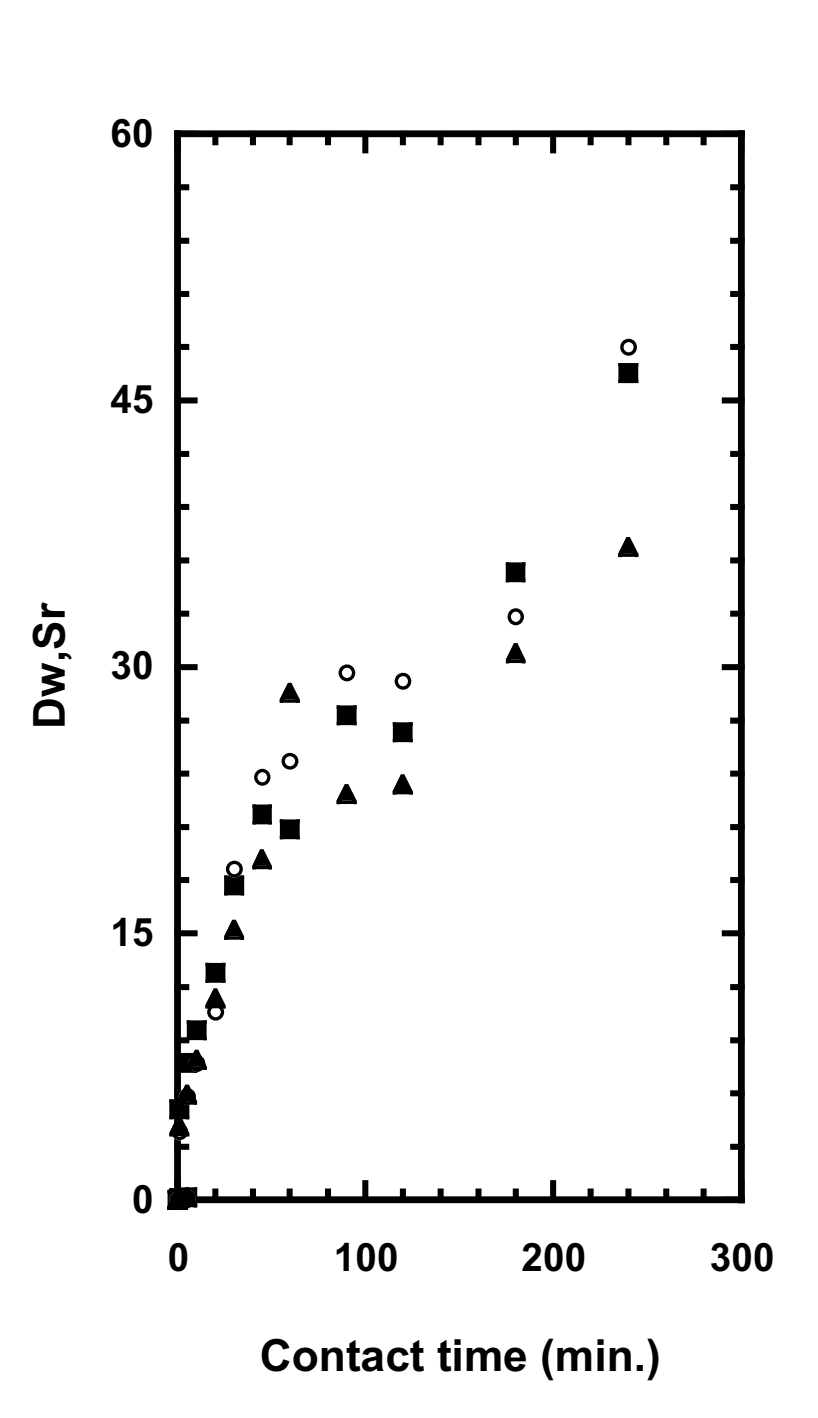

Figure 10.

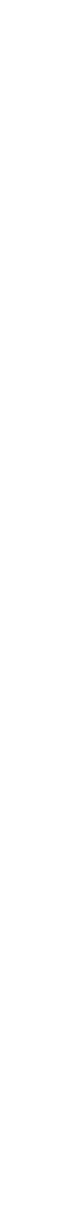

.

\author{
Figure 10.
}




\section{Graphical abstract}

\section{Crown ether-ionic liquid mixtures}

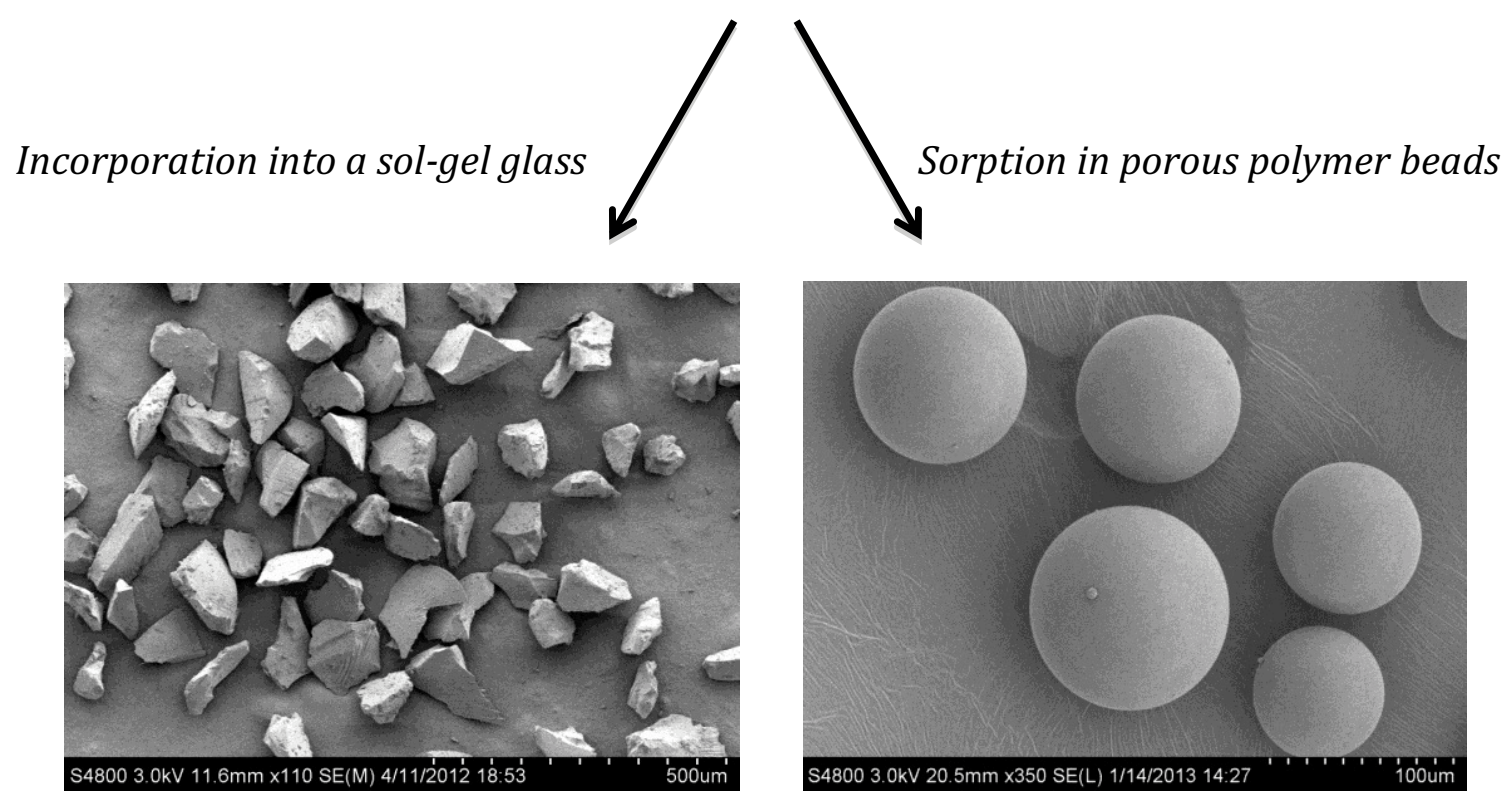

